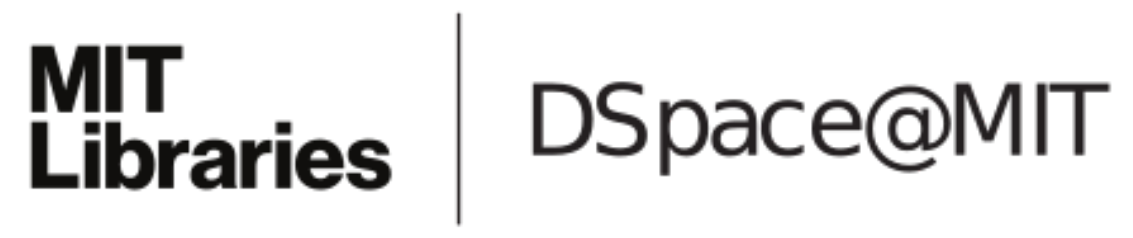

\author{
MIT Open Access Articles
}

\section{THE ATMOSPHERES OF EARTHLIKE PLANETS AFTER GIANT IMPACT EVENTS}

The MIT Faculty has made this article openly available. Please share how this access benefits you. Your story matters.

Citation: Lupu, R. E., Kevin Zahnle, Mark S. Marley, Laura Schaefer, Bruce Fegley, Caroline Morley, Kerri Cahoy, Richard Freedman, and Jonathan J. Fortney. "THE ATMOSPHERES OF EARTHLIKE PLANETS AFTER GIANT IMPACT EVENTS.” The Astrophysical Journal 784, no. 1 (February 28, 2014): 27. (C) 2014 The American Astronomical Society

As Published: http://dx.doi.org/10.1088/0004-637x/784/1/27

Publisher: IOP Publishing

Persistent URL: http://hdl.handle.net/1721.1/92946

Version: Final published version: final published article, as it appeared in a journal, conference proceedings, or other formally published context

Terms of Use: Article is made available in accordance with the publisher's policy and may be subject to US copyright law. Please refer to the publisher's site for terms of use. 


\title{
THE ATMOSPHERES OF EARTHLIKE PLANETS AFTER GIANT IMPACT EVENTS
}

\author{
R. E. Lupu ${ }^{1}$, Kevin Zahnle ${ }^{2}$, Mark S. Marley ${ }^{2}$, Laura Schaefer ${ }^{3}$, Bruce Fegley ${ }^{4}$, Caroline Morley $^{5}$, \\ Kerri CahoY $^{6}$, Richard FreEdman ${ }^{1}$, And Jonathan J. Fortney ${ }^{5}$ \\ ${ }^{1}$ SETI Institute/NASA Ames Research Center, Moffet Field, CA 94035, USA; Roxana.E.Lupu@ @asa.gov \\ 2 NASA Ames Research Center, Moffet Field, CA 94035, USA \\ ${ }^{3}$ Harvard/Smithsonian Center for Astrophysics, Cambridge, MA 02138, USA \\ ${ }^{4}$ Planetary Chemistry Laboratory, Department of Earth \& Planetary Sciences and \\ McDonnell Center for the Space Sciences, Washington University, St. Louis, MO 63130, USA \\ ${ }^{5}$ Department of Astronomy and Astrophysics, University of California, Santa Cruz, CA 95064, USA \\ ${ }^{6}$ Massachusetts Institute of Technology, Cambridge, MA 02139, USA \\ Received 2013 August 2; accepted 2014 January 14; published 2014 February 28
}

\begin{abstract}
It is now understood that the accretion of terrestrial planets naturally involves giant collisions, the moon-forming impact being a well-known example. In the aftermath of such collisions, the surface of the surviving planet is very hot and potentially detectable. Here we explore the atmospheric chemistry, photochemistry, and spectral signatures of post-giant-impact terrestrial planets enveloped by thick atmospheres consisting predominantly of $\mathrm{CO}_{2}$ and $\mathrm{H}_{2} \mathrm{O}$. The atmospheric chemistry and structure are computed self-consistently for atmospheres in equilibrium with hot surfaces with composition reflecting either the bulk silicate Earth (which includes the crust, mantle, atmosphere, and oceans) or Earth's continental crust. We account for all major molecular and atomic opacity sources including collision-induced absorption. We find that these atmospheres are dominated by $\mathrm{H}_{2} \mathrm{O}$ and $\mathrm{CO}_{2}$, while the formation of $\mathrm{CH}_{4}$ and $\mathrm{NH}_{3}$ is quenched because of short dynamical timescales. Other important constituents are $\mathrm{HF}, \mathrm{HCl}$, $\mathrm{NaCl}$, and $\mathrm{SO}_{2}$. These are apparent in the emerging spectra and can be indicative that an impact has occurred. The use of comprehensive opacities results in spectra that are a factor of two lower brightness temperature in the spectral windows than predicted by previous models. The estimated luminosities show that the hottest post-giant-impact planets will be detectable with near-infrared coronagraphs on the planned $30 \mathrm{~m}$ class telescopes. The $1-4 \mu \mathrm{m}$ will be most favorable for such detections, offering bright features and better contrast between the planet and a potential debris disk. We derive cooling timescales on the order of $10^{5-6} \mathrm{yr}$ on the basis of the modeled effective temperatures. This leads to the possibility of discovering tens of such planets in future surveys.
\end{abstract}

Key words: brown dwarfs - planetary systems - planets and satellites: general - radiative transfer - stars: low-mass

Online-only material: color figures

\section{INTRODUCTION}

The final assembly of terrestrial planets is now universally thought to have occurred through a series of giant impacts-essentially collisions between planets-spread out over some 100 million years (Cameron \& Ward 1976; Wetherill 1985; Lissauer 1993; Chambers 2004; Raymond et al. 2004). The most famous of these is Earth's own moon-forming impact (Hartmann et al. 1986; Benz et al. 1986; Canup \& Righter 2000; Canup \& Asphaug 2001; Agnor \& Asphaug 2004; Canup 2004). It takes at least 10 collisions between planets to make a Venus and an Earth, as not every collision results in a merger. In the aftermath of one of these collisions, the surviving planet is hot and can remain hot for millions of years (Zahnle 2006; Zahnle et al. 2007). During this phase of accretion, a prototerrestrial planet may have a dense steam atmosphere (e.g., Abe \& Matsui 1988; Matsui \& Abe 1986; Zahnle et al. 1988; Hashimoto et al. 2007; Schaefer \& Fegley 2010). Eventually, the atmosphere cools and water vapor condenses into clouds. How long the stricken planet remains hot depends on the size of the collision and the nature of the planet's atmosphere. While hot, the planet will be bright, especially in the near-IR, where spectral windows reveal hotter atmospheric depths.

Such young, post-giant-impact terrestrial planets will be far brighter and easier to detect around nearby young stars than old, cold terrestrial planets (Stern 1994; Miller-Ricci et al. 2009). Furthermore, the time period after the last giant impact sets the boundary condition for the subsequent thermal evolution of Earth. Whether a terrestrial planet retains water or enters into a runaway greenhouse ultimately depends upon the conditions after the last giant impact (Hamano et al. 2013). The classic early studies of runaway greenhouse atmospheres (e.g., Ingersoll 1969) used a gray approximation for water opacity and neglected other opacity sources. Second-generation models that greatly improved on the radiative transfer in runaway and near-runaway greenhouse atmospheres were developed independently by Kasting (1988) and Abe \& Matsui (1988). These models, state-of-the-art in their day, used multiple bands of $\mathrm{H}_{2} \mathrm{O}$ and $\mathrm{CO}_{2}$, but the description of hot bands was necessarily crude given what was known at the time. Furthermore, these models completely neglected other opacity sources, some of which (e.g., the alkali metals) are now known (thanks to brown dwarf science, e.g., Burrows et al. 2000) to be of first-order importance.

The composition of a postimpact atmosphere is unlikely to be a pure mixture of $\mathrm{H}_{2} \mathrm{O}$ and $\mathrm{CO}_{2}$. At a minimum, volatile species will evaporate and the atmosphere will equilibrate with the surface. Thus, the atmospheric composition depends upon the surface composition. Whether or not the surface is oxidized $\left(\mathrm{Fe}^{3+}\right.$ minerals) versus reduced $\left(\mathrm{Fe}^{2+}\right.$ minerals), for example, will control the oxidation state of carbon compounds in the atmosphere. Thus, any model of the atmospheric structure of a postimpact world must consider a range of surface compositions. 
Radiometric dating shows that the Earth's continental crust (CC) formed very early in the history of the Earth. As noted by Fegley \& Schaefer (2012), coupled modeling of the shortlived ${ }^{182} \mathrm{Hf}-{ }^{182} \mathrm{~W}$ and ${ }^{146} \mathrm{Sm}-{ }^{142} \mathrm{Nd}$ systems by Moynier et al. (2010) shows Earth's crust formed 38-75 million years after the formation of the solar system. The oldest dated crustal samples are detrital zircons from Jack Hills, Australia, which are up to 4404 million years old (Wilde et al. 2001; Harrison et al. 2005). These zircons show that CC existed 164 million years after formation of the solar system. Thus, it is likely that earthlike rocky exoplanets also formed felsic crusts very early in their evolution. However, the alternative case that the surface of a postimpact world does not yet reflect the formation of continents and is composed mostly of mafic silicates, like the bulk silicate Earth (BSE), should also be considered.

In this paper we report on our calculation of the atmospheric thermal structure, equilibrium and disequilibrium chemistry, emergent spectra, and thermal evolution of post-giant-impact terrestrial planets. On the basis of these results, we also discuss the detectability of such worlds and the spectral markers that can discriminate between different surface chemistries. The framework we present is relevant to a variety of problems, including the runaway greenhouse and the early evolution of the Earth-Moon system. We necessarily neglect some processes that might be important, such as cloud formation and a selfconsistent photochemical model, which are deferred to a future paper.

\section{MODELING}

To model the atmospheric thermal structure of postimpact worlds, we construct a suite of radiative-convective equilibrium models that are consistent with a specified range of surface temperatures. For each model, we specify the incident flux, surface gravity and pressure, and surface chemical composition. We note that the impacted planet cools over a timescale of $10^{5}-10^{6} \mathrm{yr}$ (Zahnle et al. 2007), while the timescale for atmospheric adjustment to radiative and convective equilibrium is far shorter, in the range of a day or so. Thus, even though the atmosphere will cool through time, the thermal structure for a given surface temperature is well defined. This is the same procedure that is commonly used for evolution calculations for gas giants where a single atmospheric boundary is associated with a cooling planet or brown dwarf.

For a specified surface temperature, we solve for the radiative-convective $T-p$ profile by taking into account the heating from the surface and the incoming solar radiation, ensuring energy balance throughout the atmosphere. The atmosphere code we employ iteratively solves for radiative-convective equilibrium by adjusting the size of the convection zone until the lapse rate everywhere in the convective region is sub-adiabatic. This code was originally developed for modeling Titan's atmosphere (McKay et al. 1989) and has been extensively modified and applied to the study of brown dwarfs (e.g., Marley et al. 1996; Cushing et al. 2008; Saumon \& Marley 2008) and solar and extrasolar giant planets (Marley et al. 1999; Marley \& McKay 1999; Fortney et al. 2008a, 2008b). When fully converged, the radiative layers are typically in full radiative energy balance to about 1 part in $10^{5}$. Our solution achieves this energy balance by using a matrix method that progressively adjusts an initial guess at the $T-p$ profile (Pollack \& Ohring 1973; McKay et al. 1989) rather than by time stepping (e.g., Gierasch \& Goody 1968; Pavlov et al. 2000). Both approaches are intrin-
Table 1

Composition of Vaporized Material

\begin{tabular}{|c|c|c|}
\hline Compound & $\begin{array}{c}\text { Continental Crust }{ }^{\mathrm{a}, \mathrm{b}} \\
\text { wt. }(\%)\end{array}$ & $\begin{array}{c}\text { Bulk Silicate Earth }{ }^{\mathrm{b}, \mathrm{c}} \\
\text { wt. }(\%)\end{array}$ \\
\hline $\mathrm{SiO}_{2}$ & 64.0 & 45.9 \\
\hline $\mathrm{MgO}$ & 2.4 & 37.1 \\
\hline $\mathrm{Al}_{2} \mathrm{O}_{3}$ & 14.7 & 4.6 \\
\hline $\mathrm{TiO}_{2}$ & 0.59 & 0.22 \\
\hline $\mathrm{FeO}$ & $\cdots$ & 8.2 \\
\hline $\mathrm{Fe}_{2} \mathrm{O}_{3}$ & 4.9 & $\cdots$ \\
\hline $\mathrm{CaO}$ & 4.1 & 3.7 \\
\hline $\mathrm{Na}_{2} \mathrm{O}$ & 2.9 & 0.35 \\
\hline $\mathrm{K}_{2} \mathrm{O}$ & 3.1 & 0.03 \\
\hline $\mathrm{P}_{2} \mathrm{O}_{5}$ & 0.17 & $\cdots$ \\
\hline $\mathrm{MnO}$ & 0.08 & $\cdots$ \\
\hline \multicolumn{3}{|c|}{ Elemental breakdown } \\
\hline $\mathrm{H}$ & 0.045 & 0.006 \\
\hline $\mathrm{C}$ & 0.199 & 0.006 \\
\hline $\mathrm{N}$ & 0.006 & $0.88 \mathrm{e}-4$ \\
\hline $\mathrm{O}$ & 47.20 & 44.42 \\
\hline $\mathrm{S}$ & 0.070 & 0.027 \\
\hline $\mathrm{F}$ & 0.053 & 0.002 \\
\hline $\mathrm{Cl}$ & 0.047 & 0.004 \\
\hline $\mathrm{Si}$ & 28.80 & 21.61 \\
\hline $\mathrm{Al}$ & 7.96 & 2.12 \\
\hline $\mathrm{Fe}$ & 4.32 & 6.27 \\
\hline $\mathrm{Ca}$ & 3.85 & 2.46 \\
\hline $\mathrm{Na}$ & 2.36 & 0.29 \\
\hline $\mathrm{Mg}$ & 2.20 & 22.01 \\
\hline $\mathrm{K}$ & 2.14 & 0.02 \\
\hline $\mathrm{Ti}$ & 0.401 & 0.12 \\
\hline $\mathrm{P}$ & 0.076 & 0.008 \\
\hline $\mathrm{Cr}$ & 0.013 & 0.29 \\
\hline $\mathrm{Mn}$ & 0.072 & 0.11 \\
\hline
\end{tabular}

Notes.

a Wedepohl (1995).

b Schaefer et al. (2012).

${ }^{c}$ Fegley \& Schaefer (2012).

sically quasi-static, with the matrix method being more efficient (e.g., Ramanathan \& Coakley 1978).

To derive the atmospheric composition, we rely on thermochemical equilibrium calculations for "earthlike" rocky planets that are heated to high temperatures (Schaefer et al. 2012). The chemistry calculations are done for a grid with temperatures from 300 to $4000 \mathrm{~K}$ and one microbar to 300 bar pressures by using a Gibbs energy minimization code (see van Zeggeren $\&$ Storey 1970). Approximately $~ 800$ compounds (solid, liquid, gas) of the elements are included in the calculations. We use two reasonable representative compositions of the silicate portion of rocky planets: (1) the Earth's CC (Wedepohl 1995) and (2) the BSE (Kargel \& Lewis 1993). These two compositions allow us to examine differences in atmospheres formed by outgassing of silica-rich (felsic) rocks-like the Earth's continental crust - and $\mathrm{MgO}$ - and $\mathrm{FeO}$-rich (mafic) rocks-like the BSE. These compositions describe the class of rocky planets around stars of solar metallicity, with a differentiated Fe metal and FeS core. For reference, the BSE is the composition of the silicate portion of the Earth that evolved into the present-day mantle, crust, oceans, and atmosphere. The mantle makes up 99.4\% by mass of the BSE; thus, the BSE composition is similar to that of Earth's mantle (Schaefer et al. 2012). The most important compounds considered in the calculations of CC and BSE compositions are listed in Table 1 . The breakdown in terms 
of the most abundant elements for the two compositions is listed in the same table.

For both compositions, we generate a grid of atmosphere models for surface pressures $p_{\text {surf }}$ of 10 and $100 \mathrm{bar}$, surface gravities $g=10$ and $30 \mathrm{~m} \mathrm{~s}^{-2}$, and spanning surface temperatures from 1000 to $2200 \mathrm{~K}$ in steps of $200 \mathrm{~K}$. The atmospheres are heated both from below, given the specified surface temperature, and from above by the incident visible flux from the Sun at 1 AU. Other stellar types, including a potential faint young Sun (appropriate for the moon-forming impact), are not considered in this work. Insolation is consistently treated as part of the energy balance in the planet's atmosphere, and it affects the resulting $T-p$ profile. As shown in Section 3.1, in the runaway greenhouse limit the incident stellar flux sets the lowest surface temperature the planet can attain. Outside this limit, we check the effect of the incident radiation on the effective temperature of the planet by varying the star-planet distance and find it to be small (see Figure 2).

Although condensation is accounted for in the chemical equilibrium abundances and gas opacities, we neglect here the opacity of cloud-forming species and we do not compute a wet adiabat because water condensation generally does not occur in the model tropospheres. In our models, the most important condensing species is $\mathrm{NaCl}$. Most Fe metal remains in the magma and is never vaporized in our models (Schaefer et al. 2012); therefore, Fe metal condensation is not an issue. We estimate that the effects of the latent heat released by the condensation of $\mathrm{NaCl}$ on the adiabatic lapse rate is negligible compared with the gas $\mathrm{C}_{p}$. For a condensing species, the effect of the latent heat on the lapse rate is given by the term $L X_{i}\left(L / R T^{2}-1 / T\right)$, where $L$ is the latent heat, $R$ is the ideal gas constant, and $X_{i}$ is the mole fraction of the condensing species (Lewis 1969). At $1600 \mathrm{~K}$, the enthalpy of reaction for $\mathrm{NaCl}$ (gas) $=\mathrm{NaCl}$ (liquid) is $-170,217 \mathrm{~J} \mathrm{~mol}^{-1}$, and the corresponding heat effect will be $1.25 \mathrm{~J} \mathrm{~mol}^{-1} \mathrm{~K}$, where $X_{i}$ for $\mathrm{NaCl}$ is 0.001 . This effect from the condensing $\mathrm{NaCl}$ is only $2.4 \%$ of the $\mathrm{C}_{p}$ of the gas itself, which is about 53.47 $\mathrm{J} \mathrm{mol}^{-1} \mathrm{~K}$ (for a 50:50 $\mathrm{CO}_{2}-\mathrm{H}_{2} \mathrm{O}$ mixture). We conclude that the departures from the dry adiabat are insignificant for the cases presented in this paper.

The radiative transfer equations for incoming solar radiation are solved using the delta-discrete ordinates method of Toon et al. (1989), while thermal radiative transfer is computed using the two-stream source function method, also of Toon et al. (1989). With a few exceptions, our model planets are assumed to be located at $1 \mathrm{AU}$ from the sun, and a current-day solar spectrum is used as the external radiation flux.

The radiative transfer calculation treats opacity with the correlated- $k$ technique (Goody et al. 1989) for computational speed, with 196 wavelength intervals over the whole spectrum. The correlated- $k$ coefficients are derived using 33 individual molecular opacity sources plus alkali metals, as listed in Table 2. Hot line lists are used for every species for which they are available as described in Freedman et al. (2008). In addition, we extend the opacities mentioned in Freedman et al. by using additional HITRAN data (Rothman et al. 2009). For completeness, the literature resources for the molecular opacities used in this paper are also summarized in Table 2. The listed $\mathrm{H}_{2} \mathrm{O}$ and $\mathrm{CO}_{2}$ databases are in agreement with HITEMP 2010 (Rothman et al. 2010) for the range of temperatures and pressures relevant to this paper. Our molecular and atomic opacity database is adequate for temperatures up to a few thousand degrees, as checked against the latest laboratory experiments, and is used as a validation tool for state-of-theart theoretical calculations. It has also been used for modeling the spectra of brown dwarfs with effective temperatures between 500 and $2400 \mathrm{~K}$, with excellent agreement with the observations (e.g., Cushing et al. 2008; Saumon \& Marley 2008).

The opacities for each molecule are precomputed at high spectral resolution on a 1060-point pressure-temperature grid, from 75 to $4000 \mathrm{~K}$ and $10^{-6}$ to 300 bar. The molecular and alkali chemical equilibrium abundances for each of the two compositions are interpolated on the same 1060-point $(p, T)$ grid and are used to pre-mix the opacities before deriving the $k$-coefficients. The abundances were extrapolated below $300 \mathrm{~K}$ by requiring that the partial pressure of water vapor not exceed the saturated vapor pressure at the given temperature.

In addition to the opacity sources included in the $k$ coefficients, the radiative transfer takes into account the opacity arising from collisionally induced absorption (CIA) by $\mathrm{H}_{2}$ (Freedman et al. 2008, and references therein), as well as other CIA opacity for molecules recently available through the HITRAN database (Richard et al. 2012, and references therein), i.e., $\mathrm{CH}_{4}, \mathrm{CO}_{2}, \mathrm{~N}_{2}$, and $\mathrm{O}_{2}$. The reflected part of the spectrum is accounted for by Rayleigh scattering by $\mathrm{H}_{2}, \mathrm{CH}_{4}, \mathrm{~N}_{2}, \mathrm{H}_{2} \mathrm{O}, \mathrm{CO}$, $\mathrm{CO}_{2}$, and $\mathrm{O}_{2}$, with the corresponding refractive indices (for the scattering cross sections) given by Weber (2003). All models are cloud-free. Cloudy models will be addressed in future work.

The mean molecular mass and heat capacity are also precomputed for each composition on the 1060-layer $(p, T)$ grid before being used in the radiative-convective calculation. The mean heat capacities are approximated by using available data for $\mathrm{H}_{2} \mathrm{O}, \mathrm{CO}_{2}, \mathrm{~N}_{2}, \mathrm{CO}, \mathrm{CH}_{4}, \mathrm{O}_{2}$, and $\mathrm{H}_{2}$ and by performing a mass weighted average assuming these molecules are the only constituents.

The parameter space used is similar to the one used by MillerRicci et al. (2009). By contrast, our atmospheric composition is computed self-consistently from the equilibrium chemistry of vapor in contact with a magma ocean, rather than assuming preset abundances for the entire atmosphere. The opacities for all species are used, to the extent that they are available, resulting in a fully non-gray atmosphere. We also explore the effects of disequilibrium chemistry and photochemistry, which have not been taken into account by the study of Miller-Ricci et al. (2009). This work for the first time quantifies the connection between the surface and effective temperatures, thus allowing estimates of the cooling time of post-giant-impact planets. This will enable further understanding of the early surface temperature evolution of the Earth, in the context of a non-gray atmosphere.

Atmospheric composition as a function of altitude is shown for a few representative models in Figures 4 and 5. The influence of each molecule shown is accounted for in both the radiative transfer calculation and the underlying chemistry. Emergent high-resolution spectra are computed by using the line-by-line radiative transfer code DISORT (Stamnes et al. 1988), employing a fine opacity grid at every point in the atmosphere. DISORT can take into account an external illumination source, the solar spectrum in this case, and a Lambertian lower boundary. The effects of vertical mixing and photochemistry on the atmospheric structure are discussed in Section 4.

\section{RESULTS}

\subsection{Pressure-Temperature Profiles}

Pressure-temperature profiles for all 56 models are shown in Figure 1. The total power radiated by the planet, or the 
Table 2

Molecules Used for Opacity Calculations

\begin{tabular}{|c|c|}
\hline Molecule Name & Opacity Source(s) \\
\hline $\mathrm{C}_{2} \mathrm{H}_{2}$ & HITRAN'08 ${ }^{\mathrm{a}}$ with 2011 update \\
\hline $\mathrm{C}_{2} \mathrm{H}_{4}$ & HITRAN'08 ${ }^{\mathrm{a}}$ \\
\hline $\mathrm{C}_{2} \mathrm{H}_{6}$ & HITRAN'08 ${ }^{\mathrm{a}}$ with 2010 update; Lattanzi et al. (2011) \\
\hline $\mathrm{CaH}$ & Weck et al. $(2003 b)^{b}$ \\
\hline $\mathrm{CH}_{4}$ & Brown (2005); Strong et al. (1993); Wenger \& Champion (1998) ${ }^{\mathrm{c}}$; HITRAN'08 ${ }^{\mathrm{a}}$ isotopes \\
\hline $\mathrm{ClO}$ & HITRAN'08 ${ }^{\mathrm{a}, \mathrm{d}}$ \\
\hline $\mathrm{CO}$ & HITEMP'10'; Tipping (1976) \\
\hline $\mathrm{CO}_{2}$ & Wattson \& Rothman (1986); Dana et al. (1992); HITRAN'08a isotopes \\
\hline $\mathrm{CrH}$ & Burrows et al. (2002) \\
\hline $\mathrm{FeH}$ & Dulick et al. (2003); Hargreaves et al. (2010) \\
\hline $\mathrm{H}_{2} \mathrm{CO}$ & HITRAN'08 \\
\hline $\mathrm{H}_{2} \mathrm{O}$ & Partridge \& Schwenke (1997); Gamache et al. (1998); HITRAN'08 a isotopes \\
\hline $\mathrm{H}_{2} \mathrm{~S}$ & Kissel et al. (2002); Wattson \& Rothman (1992)—private communication; HITRAN'08a isotopes \\
\hline $\mathrm{HCl}$ & HITRAN'08 ${ }^{\mathrm{a}, \mathrm{d}}$; Toth et al. (1970) \\
\hline $\mathrm{HCN}$ & Harris et al. (2006); Harris et al. (2008); GEISA ${ }^{\mathrm{f}}$ isotopes \\
\hline $\mathrm{HF}$ & HITRAN'08 ${ }^{\mathrm{a}, \mathrm{d}} ;$ Mehrotra \& Nair (1981) \\
\hline $\mathrm{LiCl}$ & Weck et al. $(2004)^{\mathrm{b}}$ \\
\hline $\mathrm{MgH}$ & Weck et al. $(2003 a)^{b}$ \\
\hline $\mathrm{N}_{2}$ & HITRAN'08a \\
\hline $\mathrm{NH}_{3}$ & Yurchenko et al. (2011); Nemtchinov et al. (2004) \\
\hline NO & HITEMP'10 \\
\hline $\mathrm{NO}_{2}$ & HITRAN'08 ${ }^{\mathrm{a}}$ \\
\hline $\mathrm{O}_{2}$ & HITRAN'08 ${ }^{\text {a }}$ with 2009 update \\
\hline $\mathrm{O}_{3}$ & HITRAN'08 ${ }^{\mathrm{a}, \mathrm{d}}$ \\
\hline OCS & HITRAN'08 a with 2009 update \\
\hline $\mathrm{OH}$ & Kurucz $(2011)^{\mathrm{g}}$; HITRAN'08 ${ }^{\mathrm{a}}$ \\
\hline $\mathrm{PH}_{3}$ & Nikitin et al. (2009); GEISA ${ }^{\mathrm{f}}$; HITRAN'08 ${ }^{\mathrm{a}}$ \\
\hline SH & RLS code (Zare et al. 1973); Berdyugina \& Livingston (2002) \\
\hline $\mathrm{SiH}$ & Kurucz $(2011)^{\mathrm{g}}$ \\
\hline $\mathrm{SiO}$ & Langhoff \& Bauschlicher (1993); Kurucz (2011) ${ }^{\mathrm{g}}$ \\
\hline $\mathrm{SO}_{2}$ & HITRAN'08 ${ }^{\mathrm{a}}$ with 2009 update \\
\hline $\mathrm{TiO}$ & Schwenke (1998); Allard et al. (2000) \\
\hline $\mathrm{VO}$ & Alvarez \& Plez (1998) \\
\hline
\end{tabular}

Notes.

a Rothman et al. (2009); http://www.cfa.harvard.edu/hitran/updates.html

b http://www.physast.uga.edu/ugamop/

${ }^{c}$ http://icb.u-bourgogne.fr/OMR/SMA/SHTDS/STDS.html

${ }^{\mathrm{d}}$ Updates are now available in the HITRAN database. Weaker lines have been added, which would not significantly affect our results.

e Rothman et al. (2010); http://www.cfa.harvard.edu/hitran/HITEMP.html

${ }^{\mathrm{f}} \mathrm{http}: / /$ ether.ipsl.jussieu.fr/etherTypo/?id=950

$\mathrm{g}$ http://kurucz.harvard.edu/molecules.html

wavelength-integrated flux, can be expressed as $\sigma_{B} T_{\text {eff }}^{4}$, where $T_{\text {eff }}$ is the temperature of a blackbody with the same emitted power. These effective temperatures are calculated by the atmospheric structure code and are listed in Table 3. Because of the greenhouse effect, the bulk silicate earth case with $p_{\text {surf }}=100$ bar (lower right panel in Figure 1) cannot reach a surface temperature as cool as $1000 \mathrm{~K}$ (black line in other panels) at the given external radiation flux. Here the models with $T_{\text {surf }} \leqslant 1600 \mathrm{~K}$ tend to a similar effective temperature, independent of $T_{\text {surf }}$, as the atmosphere becomes optically thick. Therefore, $T_{\text {eff }}$ is completely governed by the incident radiation at the top of the atmosphere, as even without any internal heating sources the surface temperature of the 100 bar atmosphere cannot drop below $\sim 1200 \mathrm{~K}$. The incoming stellar radiation gets trapped, warming the surface up to the point where the peak of the blackbody function shifts to short enough wavelengths that the radiation is able to escape through existing opacity windows. The lowest surface temperature in this case is achieved through the formation of an isothermal layer at the bottom of the atmosphere, since the atmospheric composition does not allow for a steeper adiabat. Lower surface temperatures can only be reached for a lower incident flux, for example, at larger star-planet separations.

The $T-p$ profiles tend to become isothermal at the top of the atmosphere, where the temperature is controlled by the incident solar flux. This is more clearly shown in Figure 2. In this example, using the CC composition with $p_{\text {surf }}=10$ bar and $T_{\text {surf }}=1600 \mathrm{~K}$, models with the same surface temperature show an increase in outer layer (top of the atmosphere) temperature with decreasing star-planet distance, while the reflected part of the spectrum is more prominent at smaller separations. For the models in Figure 2, the effective temperature $T_{\text {eff }}$ remains almost constant, being determined mainly by internal heating $\left(T_{\text {surf }}\right)$ and atmospheric opacity, which control the IR part of the spectrum.

The effective temperatures in Table 3 are also plotted in Figure 3. The dependence of $T_{\text {eff }}$ on $T_{\text {surf }}$ has implications for the evolution and cooling of the planet after the giant impact, which 


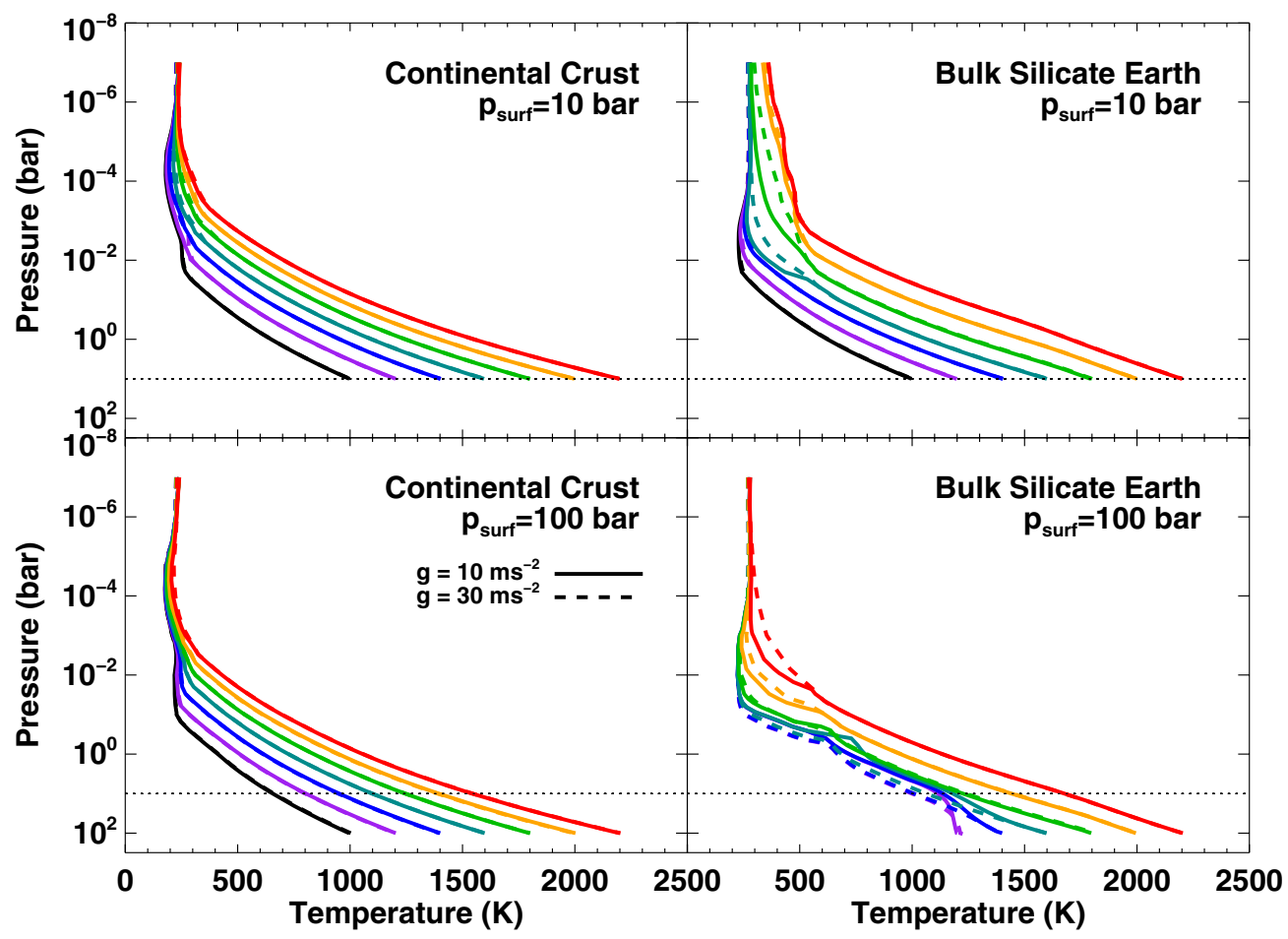

Figure 1. Pressure-temperature profiles for all models, color-coded from red to black from the highest to the lowest surface temperature. The models for the continental crust composition are shown on the left and those for the bulk silicate earth on the right. The models with $p_{\text {surf }}=10$ bar and $p_{\text {surf }}=100$ bar are shown in the upper and lower panels, respectively. A dotted line is plotted at $p_{\text {surf }}=10 \mathrm{bar}$ in all panels to guide the eye. Models with $g=30 \mathrm{~m} \mathrm{~s}^{-2}$ are shown with a dashed line. The effective temperatures for all models are listed in Table 3.

(A color version of this figure is available in the online journal.)

Table 3

Effective Temperatures

\begin{tabular}{|c|c|c|c|c|c|c|c|c|}
\hline \multirow{3}{*}{$\begin{array}{l}T_{\text {surf }} \\
(\mathrm{K})\end{array}$} & \multicolumn{4}{|c|}{ Bulk Silicate Earth } & \multicolumn{4}{|c|}{ Continental Crust } \\
\hline & \multicolumn{2}{|c|}{$10 \mathrm{~m} \mathrm{~s}^{-2}$} & \multicolumn{2}{|c|}{$30 \mathrm{~m} \mathrm{~s}^{-2}$} & \multicolumn{2}{|c|}{$10 \mathrm{~m} \mathrm{~s}^{-2}$} & \multicolumn{2}{|c|}{$30 \mathrm{~m} \mathrm{~s}^{-2}$} \\
\hline & (10 bar) & (100 bar) & (10 bar) & (100 bar) & (10 bar) & (100 bar) & (10 bar) & (100 bar) \\
\hline 1000 & 278. & $-{ }^{a}$ & 304. & $--^{a}$ & 355. & 298. & 389. & 317. \\
\hline 1200 & 323. & 265. & 356. & 266. & 409. & 315. & 451. & 340. \\
\hline 1400. & 373. & 266. & 410. & 268. & 469. & 343. & 519. & 375. \\
\hline 1600. & 413. & 267. & 487. & 278. & 531. & 380. & 589. & 419. \\
\hline 1800. & 519. & 287. & 604. & 327. & 596. & 422. & 661. & 467. \\
\hline 2000. & 647. & 353. & 723. & 424. & 661. & 467. & 734. & 515. \\
\hline 2200. & 766. & 448. & 856. & 525. & 727. & 513. & 809. & 569. \\
\hline
\end{tabular}

Notes. The header indicates the composition (bulk silicate earth vs. continental crust) and the values for $g$ (in m s${ }^{-2}$ ) and $p_{\text {surf }}$ (in bar) for each model. The surface temperature is listed in the first column. The other columns list the effective temperature, in $\mathrm{K}$, for the corresponding model. A graphical representation of this table is shown in Figure 3.

${ }^{a}$ Not available, since the BSE models with $p_{\text {surf }}=100$ bar never reach $1000 \mathrm{~K}$ surface temperature.

will be explored further in Section 5.1. Higher surface gravity models (dashed lines) have higher $T_{\text {eff }}$ for any given $T_{\text {surf }}$. This is the combined result of a decreased scale height, a different placement of convection zones, and a change in the column densities of the most important absorbers. In this case, the place where the atmosphere becomes optically thin will occur deeper in the atmosphere, at a higher pressure and temperature. Because of the blanketing effect of the atmosphere, the effective temperatures are lower than the surface temperatures by factors of a few. As is often the case in planetary atmospheres, the surface, equilibrium, effective, and wavelength-dependent brightness temperatures are not equal, and care must be taken when interpreting any relevant temperature. In Section 3.4 we compare these models to brown dwarfs of similar effective temperatures and to directly imaged exoplanets.

The flattening of the effective temperature curves at low $T_{\text {eff }}$ seen in Figure 3 is due to the atmosphere becoming optically thick, completely obscuring and shielding the surface, as the curves converge to the limit imposed by trapping of the incident flux from the star. This is the runaway greenhouse limit, in which case the surface temperature is completely decoupled from the observed effective temperature. Even without any internal energy sources, the stellar radiation trapped by the atmosphere determines the limiting $T_{\mathrm{eff}} \approx 265 \mathrm{~K}$, and for the BSE case, the surface temperature cannot cool much below $\sim 1200 \mathrm{~K}$. The radiation limit corresponding to this $T_{\text {eff }}$ is $280 \mathrm{~W} \mathrm{~m}^{-2}$, in 


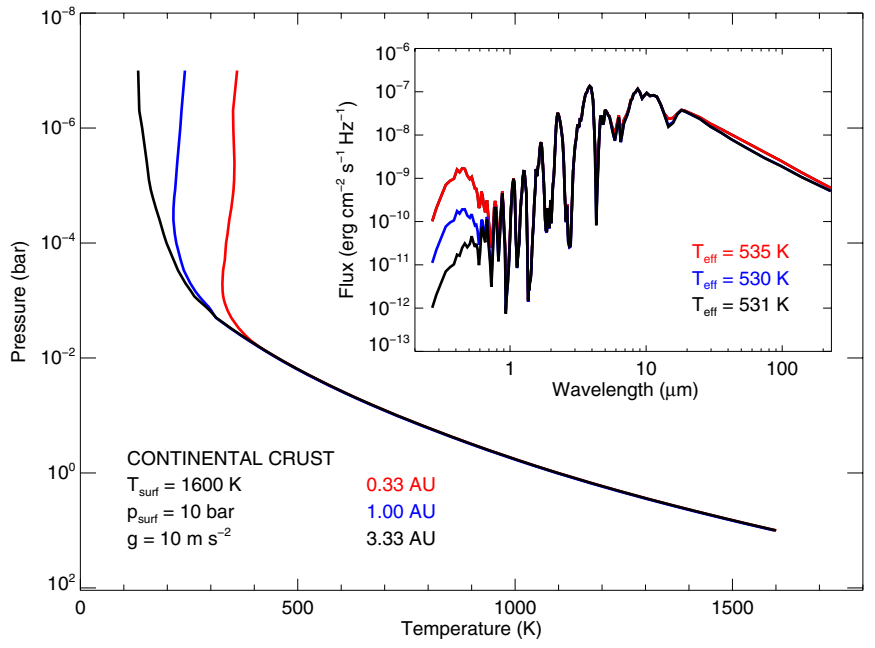

Figure 2. Variation of the atmospheric pressure-temperature profile with planet-star distance. For this the continental crust model with $p_{\text {surf }}=10 \mathrm{bar}$, $T_{\text {surf }}=1600 \mathrm{~K}$, and $g=10 \mathrm{~m} \mathrm{~s}^{-2}$ was considered. The star-planet distance controls the temperature at the top of the atmosphere, with the coldest corresponding to the largest distance. The reflected light portion of the spectrum (see insert) will also be weakest for the largest distance because of the decrease in the received flux. The effective temperature only varies by about $5 \mathrm{~K}$ between these models.

(A color version of this figure is available in the online journal.)

line with other independent model predictions for the Earth's atmosphere (Kopparapu et al. 2013; Goldblatt et al. 2012).

\subsection{Atmospheric Composition}

The relative abundances of all molecular and atomic species can be computed at any point in the atmosphere, interpolating the initial equilibrium chemistry grid. Representative abundance profiles are shown in the left panels of Figures 4 and 5 for the $\mathrm{CC}$ and bulk silicate earth cases, respectively, with $T_{\text {surf }}=1600 \mathrm{~K}, p_{\text {surf }}=100 \mathrm{bar}$, and $g=10 \mathrm{~m} \mathrm{~s}^{-2}$. The right panels of these figures show the effects of vertical mixing and photochemistry, which we discuss in more detail in Section 4. The pressure-temperature profiles for these models are shown by the dashed lines in the same figures, with the temperature axis at the top. For the $\mathrm{CC}$ case, the atmosphere is dominated by $\mathrm{H}_{2} \mathrm{O}$ and $\mathrm{CO}_{2}$, the latter becoming most abundant above the water condensation zone. The $\mathrm{H}_{2}$ and $\mathrm{CH}_{4}$ are secondary constituents in the upper atmosphere, while in the lower atmosphere they are replaced by $\mathrm{HF}, \mathrm{HCl}$, and $\mathrm{SO}_{2}$. For the bulk silicate earth composition in equilibrium with the hot magma, $\mathrm{H}_{2} \mathrm{O}$ and $\mathrm{CO}_{2}$ are dominant at higher pressures, while the upper atmosphere is composed mostly of $\mathrm{H}_{2}, \mathrm{CH}_{4}$, and $\mathrm{NH}_{3}$. While still important, $\mathrm{HCl}, \mathrm{HF}$, and $\mathrm{SO}_{2}$ are less prominent than in the $\mathrm{CC}$ case, while $\mathrm{NH}_{3}$ becomes an important opacity source. The $\mathrm{NH}_{3}$ and $\mathrm{CH}_{4}$ curves are shown as dashed because the formation of these species becomes quenched at higher altitude, as explained in Section 4. The emerging overall picture is that in spite of a relatively cool, water-dominated spectrum, these worlds present extreme, inhospitable surface conditions, even after taking chemical disequilibrium into account.

\subsection{Emergent Spectra}

An overview of the emergent low-resolution spectra is given in Figures 6 and 7 for the $\mathrm{CC}$ and the bulk silicate earth compositions, respectively. The upper panels compare spectra from models with the same surface pressure and gravity but differ-

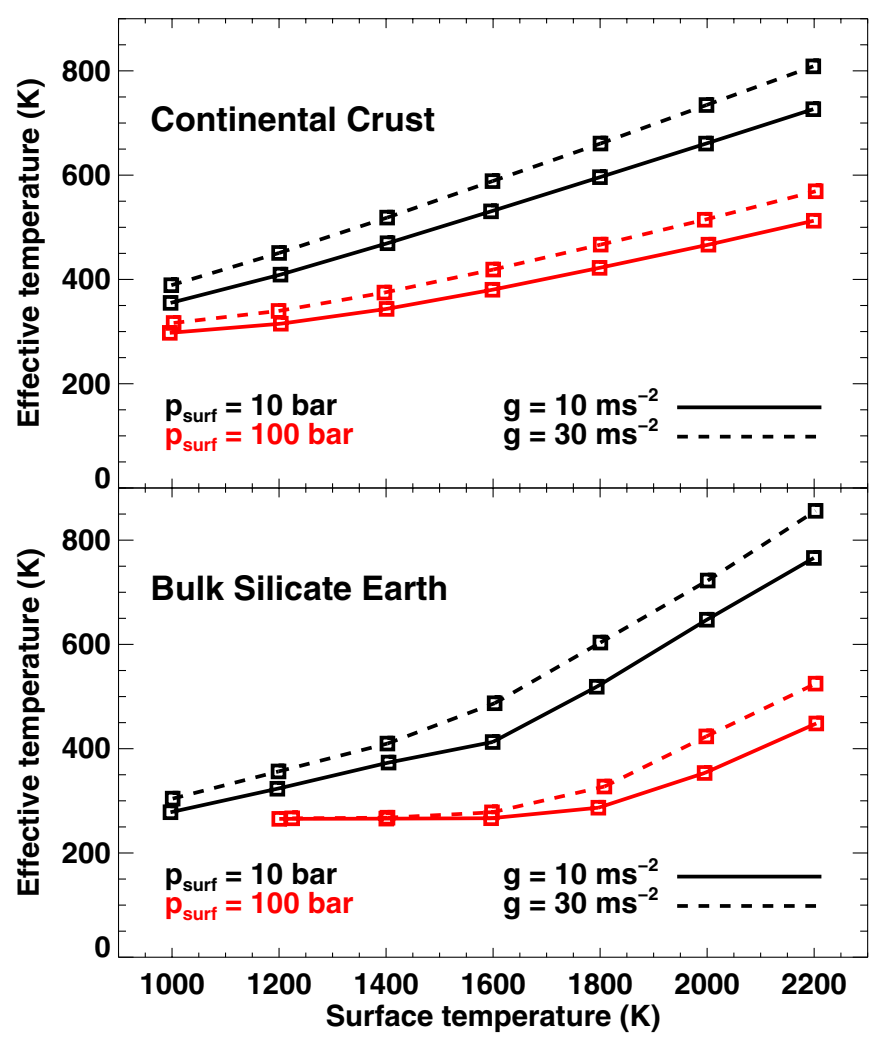

Figure 3. Effective temperature variation as a function of surface temperature for the continental crust (top panel) and bulk silicate earth (bottom panel) models. At a given surface temperature, the effective temperature is governed by both $p_{\text {surf }}$ and $g$, with larger variations for the bulk silicate earth models. The flattening of the $T_{\text {eff }}$ curves for the bulk silicate earth models with $p_{\text {surf }}=100$ bar reflects the optically thick regime where $T_{\text {eff }}$ is determined by the amount of insolation and not by the surface temperature. Effective temperature values for the models are tabulated in Table 3.

(A color version of this figure is available in the online journal.)

ent surface temperatures, while the lower panels compare the spectra for models with the same surface temperature and pressure but different surface gravities. As discussed above, models with higher $g$ have a slightly higher brightness temperature. The brightness temperature scale highlights the fact that the hot surface is obscured at virtually all wavelengths in these models. Even the brightest features in the near- to mid-IR region have a brightness temperature a factor of $\sim 2$ lower than $T_{\text {surf }}$ for the CC case, which becomes a factor of $\sim 3$ for the bulk silicate earth models. The spectral features change little with surface temperature for the $\mathrm{CC}$ composition (Figure 6) but are progressively altered for the BSE models, as the composition changes with $T_{\text {surf }}$. For the BSE case with $p_{\text {surf }}=100$ bar (upper right panel in Figure 7 ), models with $T_{\text {surf }} \leqslant 1600 \mathrm{~K}$ lead to virtually indistinguishable spectra. This is consistent with the previous conclusions about the effective temperature and reflects the fact that the atmosphere has the same structure down to the level where the radiation is escaping from.

High-resolution spectra are obtained using the line-by-line radiative transfer code DISORT (Stamnes et al. 1988), with finely sampled opacities and equilibrium abundances at every atmospheric layer as inputs. Figures 8 and 9 show highresolution spectra for the $\mathrm{CC}$ and bulk silicate earth case, respectively, at $R \sim 500-600$ resolution. The two panels compare the spectra at constant surface pressure and gravity, for increasing surface temperatures. The most important features 


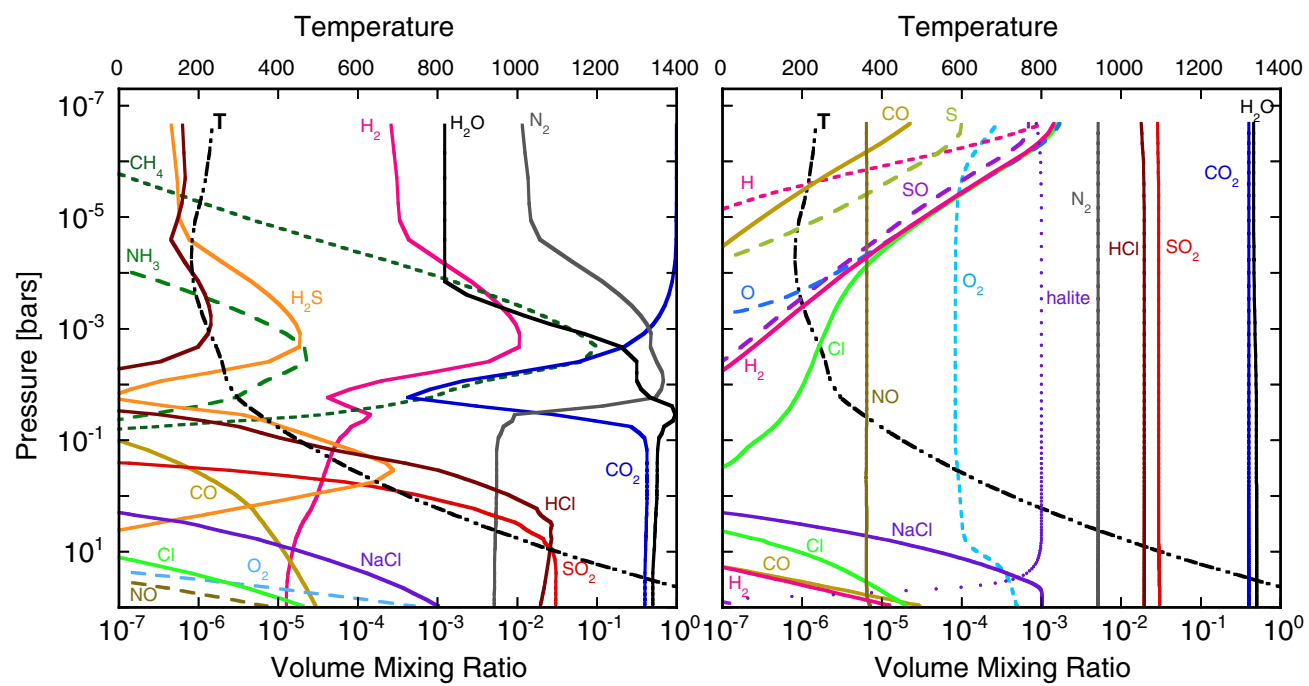

Figure 4. Chemical compositions of 100 bar atmospheres over a $1600 \mathrm{~K}$ surface in chemical equilibrium with the continental crust composition. The pressure-temperature profile for this model is shown with a dash-dotted black line, with the corresponding temperature axis at the top. Left: each level of the atmosphere equilibrates with the surface. Composition is sensitive to temperature. Geochemical volatiles $\mathrm{Na}, \mathrm{Cl}$, and $\mathrm{S}$ enter minerals. The profiles for $\mathrm{CH}_{4}$ and $\mathrm{NH}_{3}$ are shown with dashed lines, since their abundances become quenched at temperatures between 900 and $1000 \mathrm{~K}$ (see Section 4.1). Right: results from the photochemical kinetics model using $K_{z z}$ computed from the convective heat flux. Composition is dominated by mixing at middle altitudes and photolysis at high altitudes. Hydrochloric acid, salt (halite), and various sulfur species are mixed through the atmosphere.

(A color version of this figure is available in the online journal.)

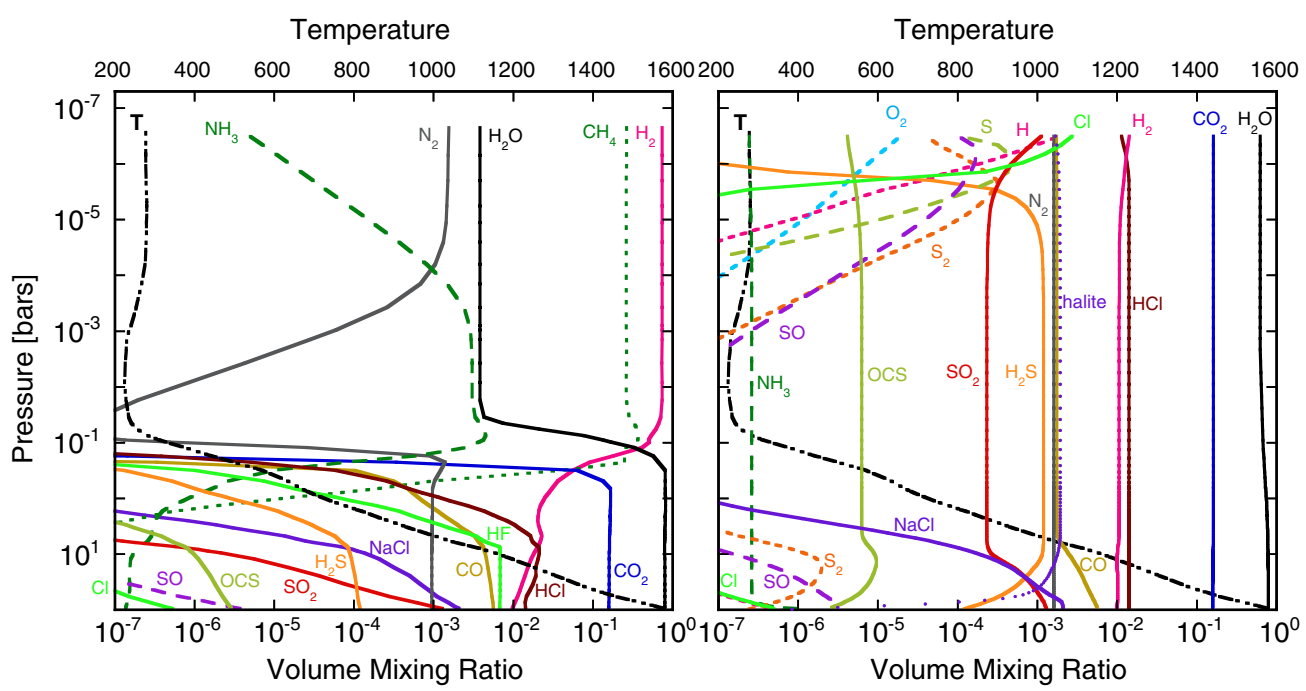

Figure 5. Same as Figure 4 for the bulk silicate earth case. Because of quenching of $\mathrm{CH}_{4}$ and $\mathrm{NH}_{3}$ reactions, these species are no longer important in this scenario (left panel). The atmospheric composition will resemble more closely the one for the continental crust case, dominated by water and $\mathrm{CO}_{2}$. However, more sulfur species are produced by equilibrium chemistry in the lower atmosphere than in the continental crust case and are subsequently mixed in the higher layers.

(A color version of this figure is available in the online journal.)

are identified. As expected from the atmospheric composition, in the $\mathrm{CC}$ case the spectrum is dominated by $\mathrm{H}_{2} \mathrm{O}$ and $\mathrm{CO}_{2}$. As the surface temperature decreases, $\mathrm{CH}_{4}$ features become more prominent, while the $\mathrm{CO}_{2}$ features weaken. The Na I D lines at $0.59 \mu \mathrm{m}$ are apparent at the highest $T_{\text {surf }}$ and low $p_{\text {surf }}$ (upper panel). The spectra of BSE models (see Figure 9) are also dominated by water absorption. However, in contrast to the $\mathrm{CC}$ case, the $\mathrm{CO}_{2}$ contribution is much diminished, being only significant for the higher $T_{\text {surf }}$ models, while $\mathrm{CH}_{4}$ becomes more important, comparable to water at lower temperatures for $p_{\text {surf }}=10$ bar and especially predominant in all models for $p_{\text {surf }}=100$ bar. Interestingly, under the assumption of equilibrium chemistry, $\mathrm{NH}_{3}$ is a significant absorber and an abundant molecule for the BSE case, with lines that become completely optically thick for most of the $p_{\text {surf }}=100 \mathrm{bar}$ models, and for low $T_{\text {surf }}$ in general. The combination of $\mathrm{H}_{2} \mathrm{O}$, $\mathrm{CH}_{4}$, and $\mathrm{NH}_{3}$ lines covering most of the IR region makes these planets heavily obscured and subject to a strong greenhouse effect. For emphasis, in Figure 10 we compare directly the BSE and CC spectra shown in Figures 9 and 8. These figures clearly display the aforementioned differences between the $\mathrm{CC}$ and the bulk silicate earth models. They also emphasize the similarity of the two compositions for $p_{\text {surf }}=10 \mathrm{bar}$, in which case the equilibrium chemistry for BSE does not favor significant production of $\mathrm{CH}_{4}$ and $\mathrm{NH}_{3}$, while the presence of larger amounts of $\mathrm{CO}_{2}$ and $\mathrm{SO}_{2}$ characterizes the $\mathrm{CC}$ spectrum.

The lower atmosphere constituents mentioned in Section 3.2 also have discernable features in the high-resolution spectra, 


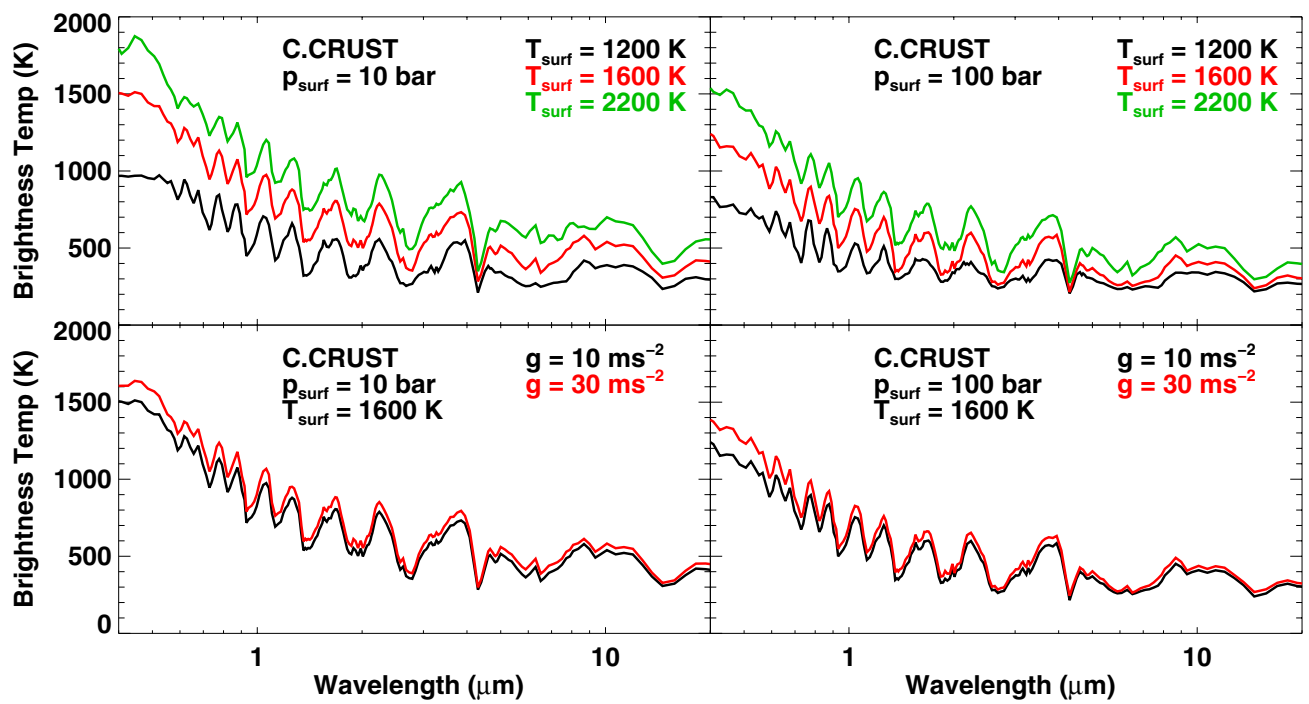

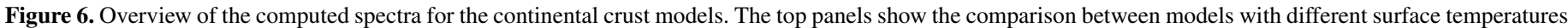

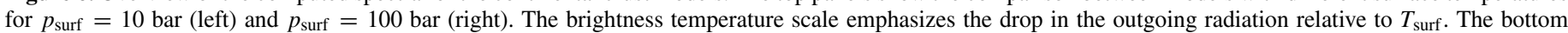

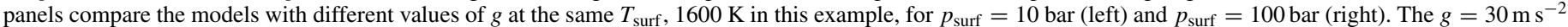
models are slightly brighter than their $g=10 \mathrm{~m} \mathrm{~s}^{-2}$ equivalents. For the individual labeling of major features in these spectra, see Figure 8 .

(A color version of this figure is available in the online journal.)

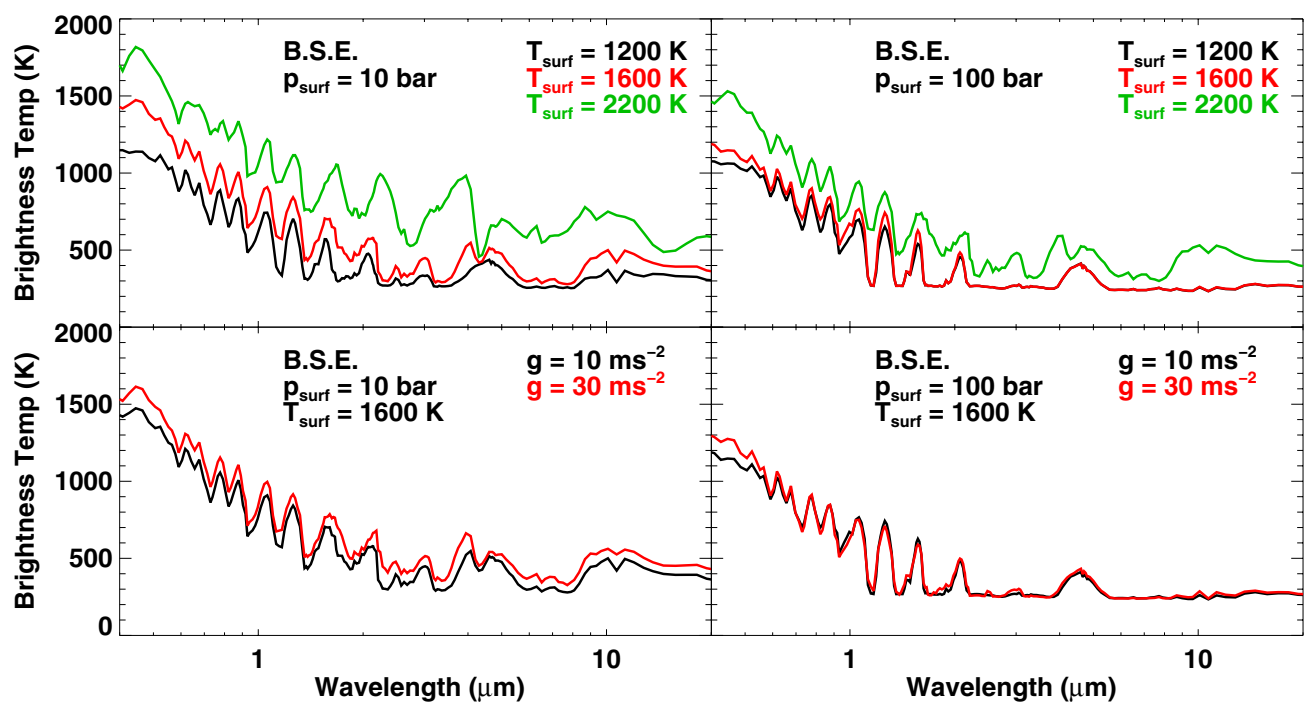

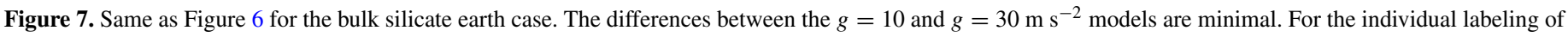
major features in these spectra, see Figure 9.

(A color version of this figure is available in the online journal.)

as some strong transitions fall in the window regions. The $\mathrm{HF}$ signature is shown in red in Figure 11, while the $\mathrm{HCl}$ and $\mathrm{SO}_{2}$ features are shown in green and blue, respectively, in Figure 12. As these constituents become prominent only at high temperatures, we show only the spectra for $T_{\text {surf }}=2200 \mathrm{~K}$. The spectra shown in color are computed after the opacity of the corresponding molecule has been removed from the mix. This helps guide the eye to the relative importance of the $\mathrm{HF}, \mathrm{HCl}$, and $\mathrm{SO}_{2}$ features, given the abundance of overlapping lines present in these spectra. For HF we distinguish a band edge at $0.867 \mu \mathrm{m}$ and a strong line pattern between 1.25 and $1.29 \mu \mathrm{m}$. $\mathrm{HCl}$ shows a similar pattern between 3.5-3.9 $\mu \mathrm{m}$, while $\mathrm{SO}_{2}$ has broadband signatures, most notably one starting at $3.95 \mu \mathrm{m}$, near the wing of the $\mathrm{CO}_{2}$ band (for context, see Figure 10). From Figure 12 it is apparent that $\mathrm{SO}_{2}$ can be considered as a marker for the $\mathrm{CC}$ composition and could be used to distinguish between a post-giant-impact planet and a cool water world. The HF and $\mathrm{HCl}$ lines are also weaker in the bulk silicate earth models.

We note that $\mathrm{HCl}$ and $\mathrm{HF}$ have been detected in the nearIR opacity windows at Venus (Bezard et al. 1990). These molecules appear to be robust indicators of a hot greenhouse atmosphere and could help distinguish such atmospheres from $\mathrm{CO}_{2}$-dominated atmospheres with cooler surface temperatures. The chemistry models of Schaefer \& Fegley (2011) predict the presence of important amounts of $\mathrm{HCl}$ and $\mathrm{HF}$ in the atmospheres of hot rocky planets and show that a cooler surface leads to less $\mathrm{HCl}$. By calculating the $T-p$ profiles, we find that these molecules are also potentially detectable in exoplanetary atmospheres.

Finally, we compare the current models to the previous result of Miller-Ricci et al. (2009) in Figure 13. All models in this figure have $p_{\text {surf }}=10 \mathrm{bar}, T_{\text {surf }}=1600 \mathrm{~K}$, 


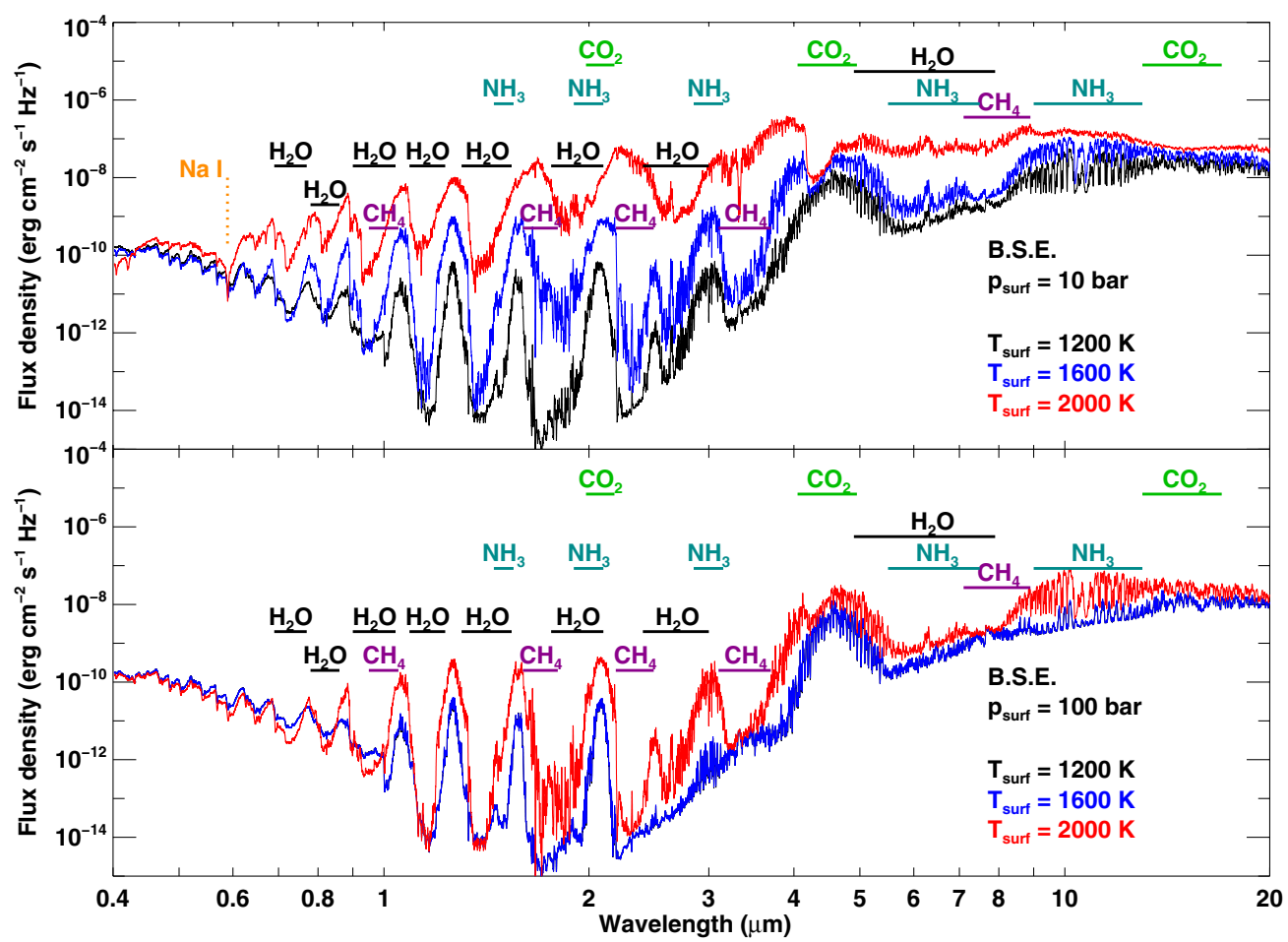

Figure 8. Comparison between the high-resolution spectra for the continental crust models as a function of surface temperature, for $p_{\text {surf }}=10$ bar (top panel) and $p_{\text {surf }}=100$ bar (bottom panel). In all cases, the dominant features remain comparatively unchanged, with the amount of $\mathrm{CH}_{4}$ increasing slowly with decreasing $T_{\text {surf }}$ and the $\mathrm{CO}_{2}$ contribution decreasing relative to water.

(A color version of this figure is available in the online journal.)

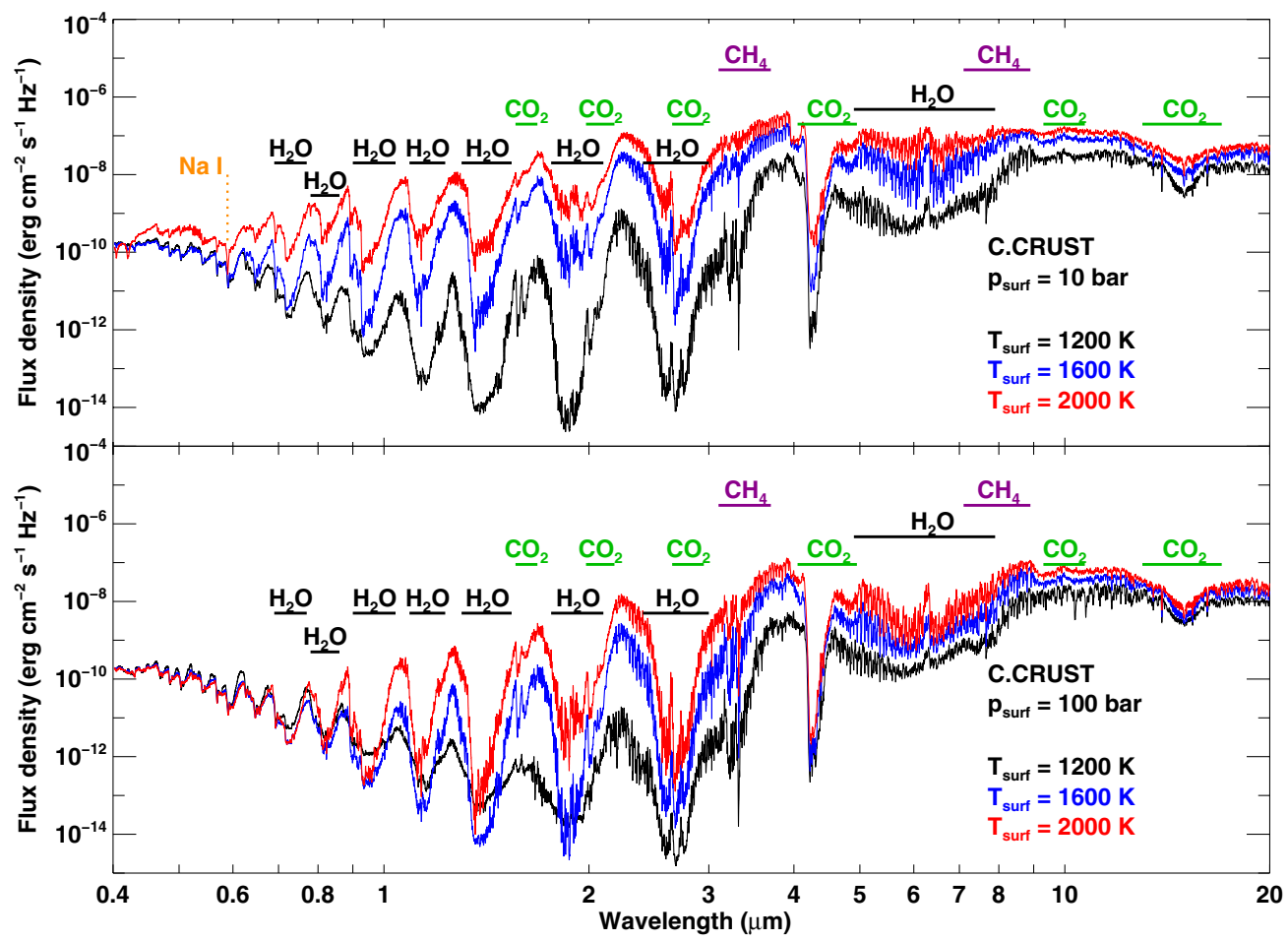

Figure 9. Same as Figure 8 for the bulk silicate earth case. For $p_{\text {surf }}=10$ bar (top panel), as $T_{\text {surf }}$ decreases, the Na I $\mathrm{D}$ lines and the $\mathrm{CO}_{2}$ features become less prominent, while the $\mathrm{CH}_{4}$ and $\mathrm{NH}_{3}$ contributions increase. For $p_{\text {surf }}=100$ bar (top panel), the $\mathrm{CO}_{2}$ is only apparent at large $T_{\text {surf }}$, while $\mathrm{NH}_{3}$ becomes one of the dominant sources of opacity for lower $T_{\text {surf }}$. Note that for $p_{\text {surf }}=100$ bar and $T_{\text {surf }} \leqslant 1600 \mathrm{~K}$, the atmosphere becomes optically thick and the models cannot be further distinguished.

(A color version of this figure is available in the online journal.) 


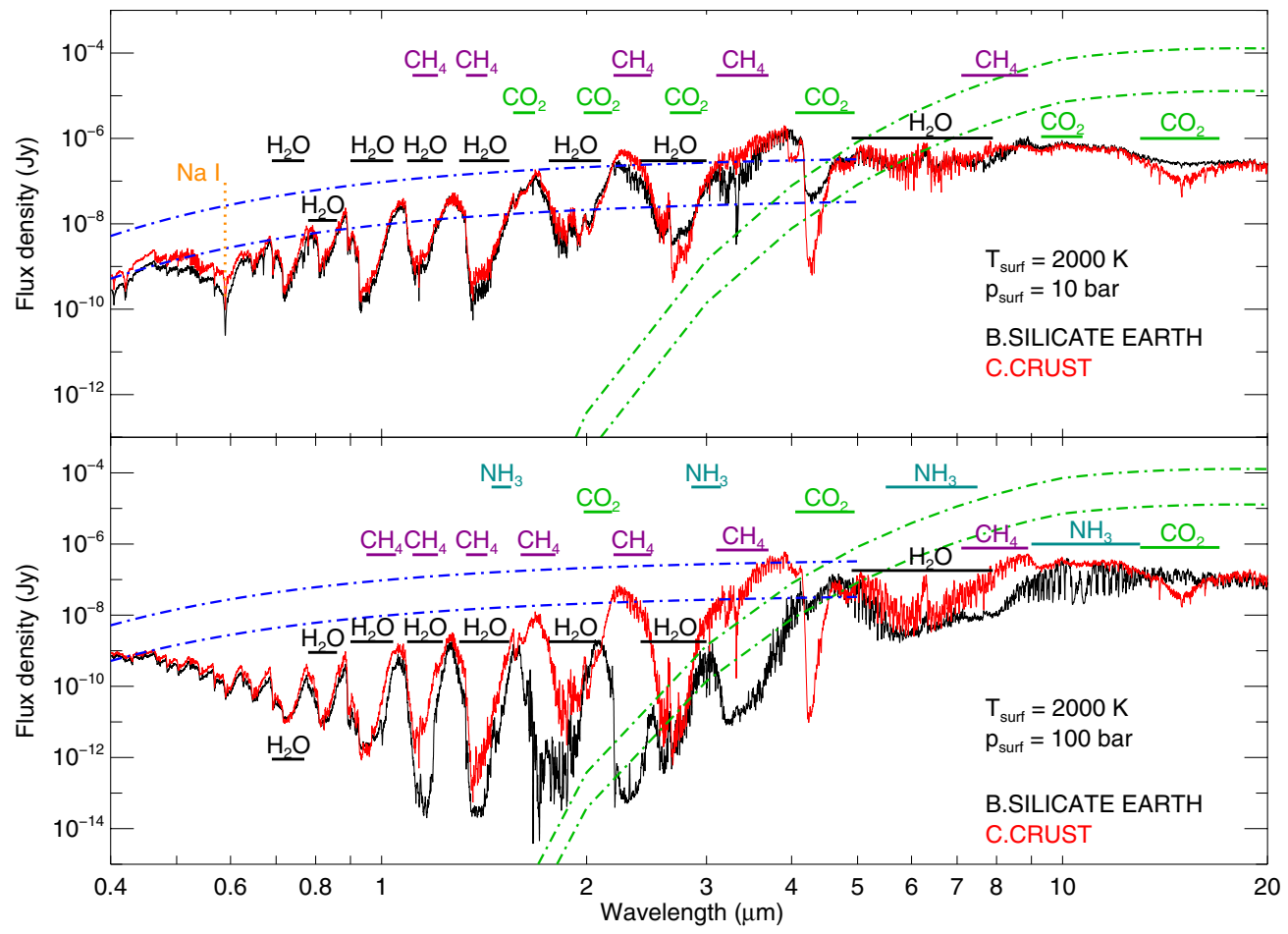

Figure 10. Comparison between the high-resolution spectra for the continental crust (red) and the bulk silicate earth (black) models, with $T_{\text {surf }}=2000 \mathrm{~K}, g=10 \mathrm{~m} \mathrm{~s}{ }^{-2}$. For the models with $p_{\text {surf }}=10$ bar (top panel), we note the stronger $\mathrm{CO}_{2}$ features (green) for the continental crust case, and the contribution of $\mathrm{CH}_{4}$ to the bulk silicate earth model. The NaI D lines (orange) are present in both cases. The bottom panel shows the same comparison for $p_{\text {surf }}=100$ bar. While the features for the continental crust model do not change considerably, the bulk silicate earth model becomes dominated by $\mathrm{CH}_{4}$ and $\mathrm{NH}_{3}$ replaces $\mathrm{CO}_{2}$ as a dominant contributor. The estimated contribution of a postimpact debris disk is shown by the dot-dashed lines. The blue and green curves represent the reflected and thermal dust emission, respectively, per resolution element of a $30 \mathrm{~m}$ telescope. The two sets of curves correspond to total luminosities of the disk of 1 and 10 zodis. The flux density is calculated at the observer, assuming a system $100 \mathrm{lt}-\mathrm{yr}$ away and a $0.8 \mathrm{AU}$ wide disk at $1 \mathrm{AU}$ from the star.

(A color version of this figure is available in the online journal.)
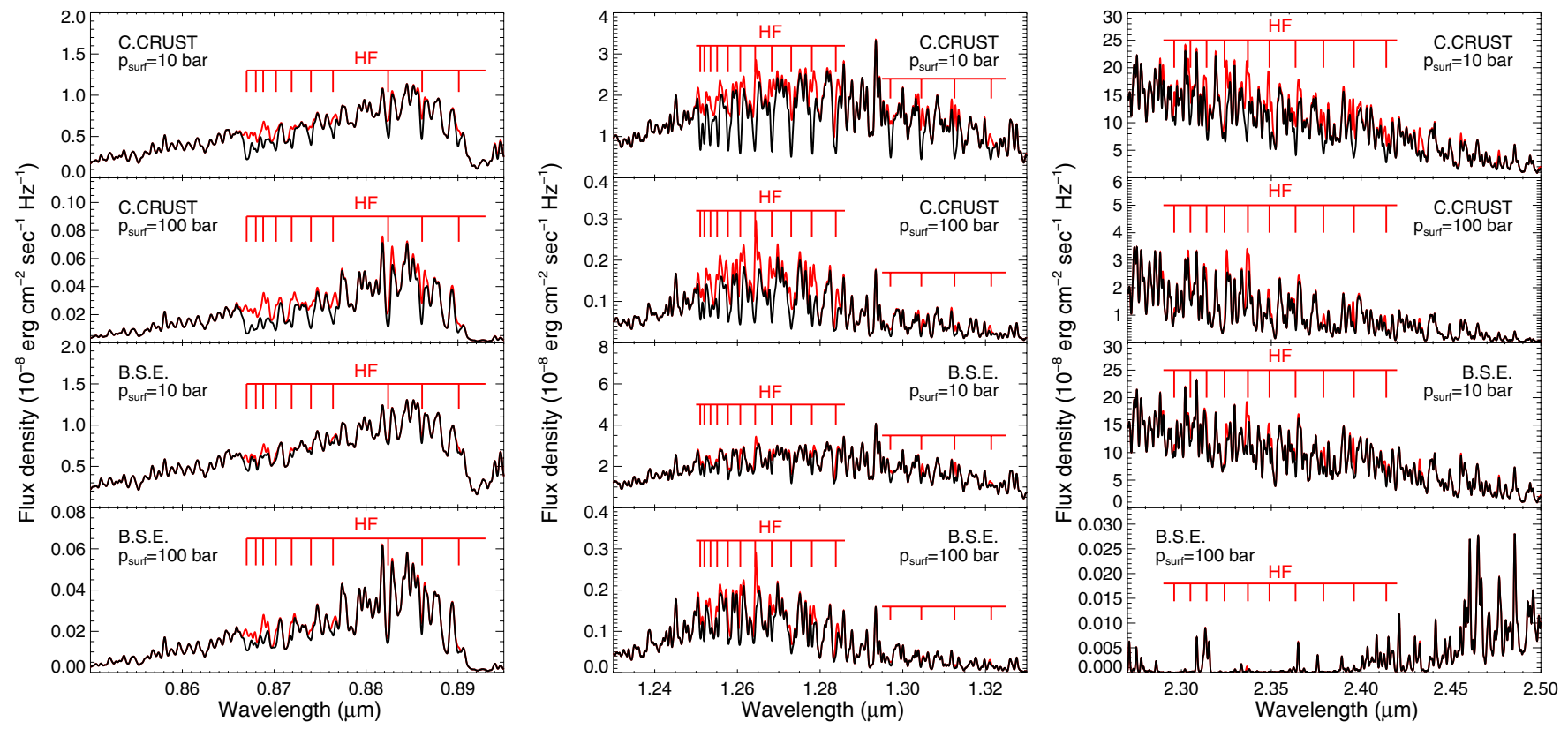

Figure 11. HF features (red) in the spectra of post-giant-impact planets, for models with a surface temperature of $2200 \mathrm{~K}$ and $g=10 \mathrm{~m} \mathrm{~s}^{-2}$. The black line shows the original high-resolution spectrum, while the red lines show the resulting high-resolution spectrum after removing the HF opacity from the mix. The HF presence is more prominent in the continental crust case.

(A color version of this figure is available in the online journal.) 

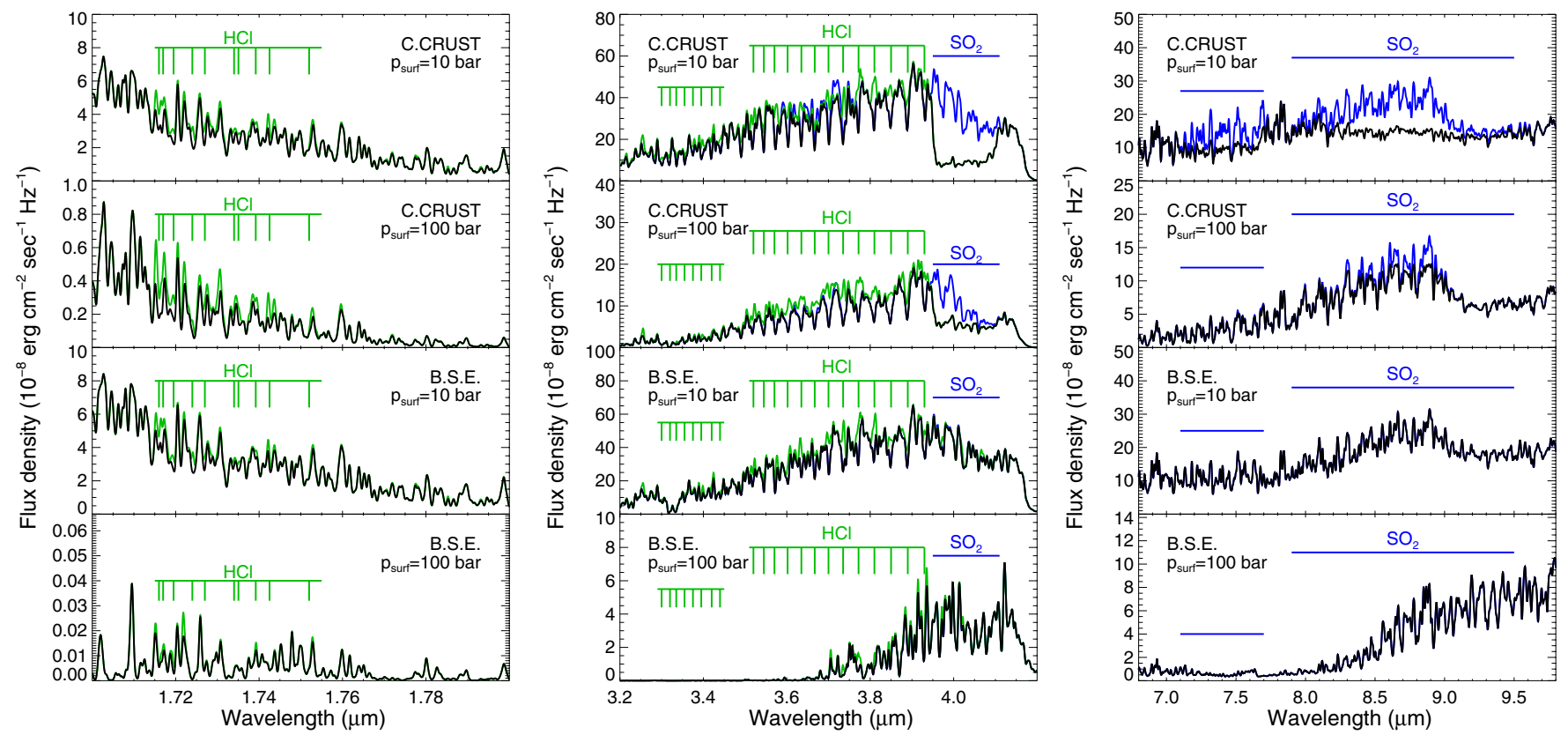

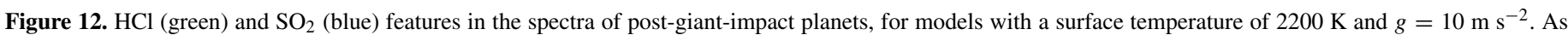

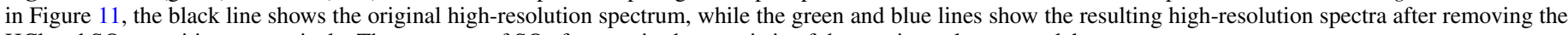
$\mathrm{HCl}$ and $\mathrm{SO}_{2}$ opacities, respectively. The presence of $\mathrm{SO}_{2}$ features is characteristic of the continental crust models.

(A color version of this figure is available in the online journal.)

and $g=10 \mathrm{~m} \mathrm{~s}^{-2}$. The Miller-Ricci et al. (2009) model contains $90 \% \mathrm{H}_{2} \mathrm{O}$ and $10 \% \mathrm{CO}_{2}$, and the predicted spectrum (black) is more similar to the $\mathrm{CC}$ model (blue), which does not contain the large $\mathrm{CH}_{4}$ absorption features present in the bulk silicate earth case (red). However, because of the additional opacity sources considered, the spectral peaks predicted by the current models have brightness temperatures one-third cooler on average than the previous estimates. The consequences for direct detection are discussed in Section 5.2.

\subsection{Model Photometry}

For a subset of the models, simulated IR photometry was calculated by using the model emergent spectra for the abundances predicted by chemical equilibrium. Absolute magnitudes were calculated in the near-IR in $Y, J, H$, and $K$ bands and in the midIR in $L^{\prime}, M^{\prime}$, Spitzer IRAC bands 1-4, WISE W1, W2, W3, and $W 4$. The radii were taken to be $1 R_{\oplus}\left(g=10 \mathrm{~m} \mathrm{~s}^{-2}\right)$ and $1.75 R_{\oplus}$ $\left(g=30 \mathrm{~m} \mathrm{~s}^{-2}\right)$, as expected for an earthlike composition (E. Lopez 2013, private communication). The model photometry is shown in Table 4.

Figure 14 shows $H-K$ color versus absolute $H$ magnitude for a selection of model postimpact Earths; observed brown dwarfs and giant planets are plotted for reference. The models with $p_{\text {surf }}=10$ bar are significantly brighter than the $p_{\text {surf }}=$ 100 bar models for similar surface temperatures. With the exception of the very hottest models that have similar colors, the $\mathrm{CC}$ composition atmospheres are significantly redder in IR color $H-K$ than the BSE composition atmospheres. This suggests that photometry alone could potentially differentiate between the compositions studied here.

It is also clear from Figure 14 that postimpact Earths are distinct in color and magnitude from brown dwarfs and giant planets; the differences are due to the larger variety of gaseous absorbers present in their atmospheres. For the hottest surface temperatures studied $(2200 \mathrm{~K})$, the absolute magnitude in the
$H$ band is roughly equal to that of the coolest brown dwarfs observed to date, but the postimpact Earths are significantly redder in IR color. This should make it possible to identify such objects from photometry alone.

\section{DISEQUILIBRIUM PROCESSES}

The abundance profiles shown in the left-hand panels of Figures 4 and 5 have been calculated assuming complete chemical equilibrium between gases, melts, and solids, i.e., the gases in each atmospheric layer are in equilibrium with each other and also with the planetary surface. In this section we consider the effects of disequilibrating processes such as vertical mixing and photochemistry on the computed model atmospheres.

\subsection{Vertical Mixing}

Sufficiently rapid vertical mixing rates relative to chemical reaction rates cause departures from chemical equilibrium in the atmospheres of gas giant planets in the solar system, extrasolar gas giant planets, and brown dwarfs (e.g., Prinn \& Barshay 1977; Fegley \& Prinn 1985, 1986, 1988; Fegley \& Lodders 1994, 1996; Lodders \& Fegley 2006). At sufficiently high temperatures and pressures in the convective regions of planetary atmospheres, chemical reactions proceed rapidly relative to vertical mixing, and the chemical lifetimes $\left(t_{\text {chem }}\right)$ are shorter than the vertical mixing times $\left(t_{\mathrm{dyn}}\right)$. In this region $t_{\text {chem }}<t_{\mathrm{dyn}}$, and chemical equilibrium is attained. As temperature and pressure decrease with increasing altitude in the convective region, reaction rates decrease exponentially and eventually to a critical altitude level at which $t_{\text {chem }}=t_{\text {dyn }}$ is reached. This critical level, which is different for each chemical reaction because of their different reaction rate constants, is the quench level at which chemical equilibrium is frozen in. At higher altitudes (and lower $T$ and $p$ ), chemical reactions proceed significantly slower and chemical lifetimes are significantly longer than vertical mixing times 


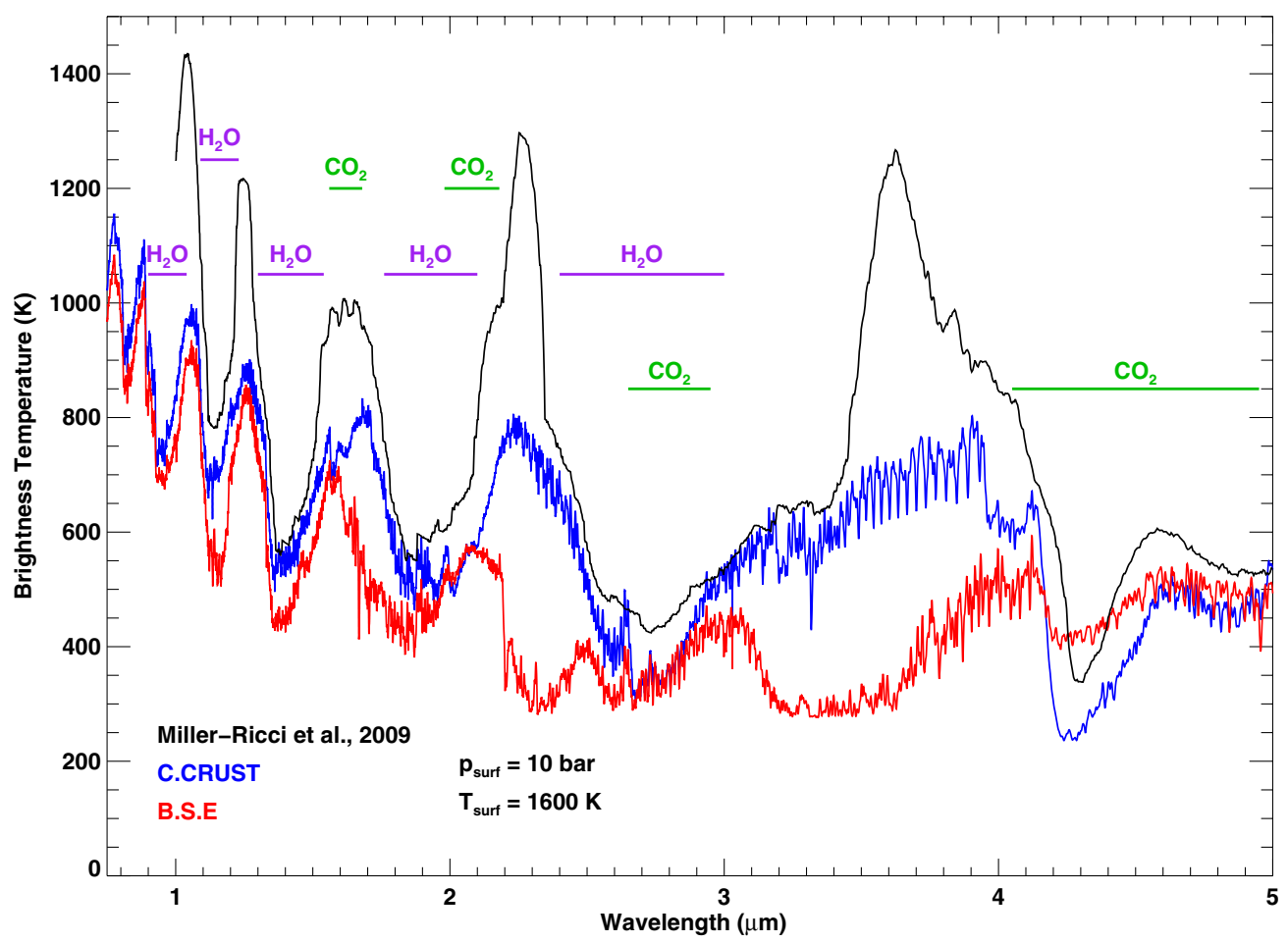

Figure 13. Comparison between the continental crust (blue), the bulk silicate earth (red), and a previous model spectrum from Miller-Ricci et al. (2009), composed of $90 \% \mathrm{H}_{2} \mathrm{O}$ and $10 \% \mathrm{CO}_{2}$. All models have roughly the same surface pressure and temperature. Because of additional opacity sources, the current models predict substantially fainter emission than the ones described in Miller-Ricci et al. (2009).

(A color version of this figure is available in the online journal.)

Table 4

Model Photometry

\begin{tabular}{|c|c|c|c|c|c|c|c|c|c|c|c|c|c|c|c|c|c|}
\hline Composition & $\begin{array}{l}\text { Pressure } \\
\text { (bar) }\end{array}$ & $\begin{array}{l}\text { Temperature } \\
\text { (K) }\end{array}$ & $\begin{array}{l}\text { Gravity } \\
\left(\mathrm{m} \mathrm{s}^{-2}\right)\end{array}$ & $Y$ & $J$ & $H$ & $K$ & $L^{\prime}$ & $M^{\prime}$ & IRAC1 & IRAC2 & IRAC3 & IRAC4 & $W 1$ & $W 2$ & $W 3$ & $W 4$ \\
\hline$\overline{\mathrm{CC}}$ & 100 & 1200 & 10 & 33.59 & 32.19 & 32.97 & 31.16 & 28.21 & 22.23 & 30.44 & 22.88 & 24.42 & 22.51 & 31.07 & 22.85 & 20.39 & 18.15 \\
\hline $\mathrm{CC}$ & 100 & 1600 & 10 & 33.44 & 32.07 & 33.0 & 31.12 & 28.13 & 22.21 & 30.41 & 22.85 & 24.39 & 22.5 & 31.06 & 22.82 & 20.36 & 18.13 \\
\hline $\mathrm{CC}$ & 100 & 2000 & 10 & 30.13 & 29.15 & 29.34 & 27.96 & 24.38 & 20.89 & 26.79 & 21.5 & 22.77 & 21.12 & 28.07 & 21.45 & 18.85 & 17.73 \\
\hline $\mathrm{CC}$ & 10 & 1200 & 10 & 32.58 & 31.33 & 32.01 & 30.26 & 25.91 & 21.68 & 28.11 & 22.22 & 23.29 & 21.43 & 29.13 & 22.16 & 19.2 & 17.76 \\
\hline $\mathrm{CC}$ & 10 & 1600 & 10 & 28.8 & 27.91 & 27.81 & 26.78 & 22.53 & 20.39 & 24.11 & 20.89 & 21.66 & 20.22 & 25.57 & 20.82 & 18.2 & 17.36 \\
\hline $\mathrm{CC}$ & 10 & 2000 & 10 & 25.73 & 24.86 & 23.48 & 21.88 & 18.87 & 19.61 & 19.44 & 19.49 & 19.04 & 18.07 & 20.17 & 19.55 & 17.2 & 16.54 \\
\hline BSE & 100 & 1000 & 10 & 40.93 & 38.29 & 35.94 & 31.54 & 23.83 & 24.16 & 24.54 & 24.37 & 25.11 & & 25.46 & 24.59 & 19.62 & 18.26 \\
\hline BSE & 100 & 1600 & 10 & 32.17 & 31.13 & 29.35 & 25.93 & 21.24 & 22.45 & 21.78 & & 23.12 & & 22.57 & 22.76 & 18.7 & 17.78 \\
\hline BSE & 100 & 2000 & 10 & 29.03 & 28.25 & 26.52 & 23.81 & 20.21 & 21.32 & 20.63 & 21.43 & 21.35 & 19.1 & 21.37 & 21.45 & 18.12 & 17.37 \\
\hline BSE & 10 & 1000 & 10 & 33.58 & 32.35 & 30.6 & 26.88 & 21.72 & 22.88 & 22.28 & 22.95 & 23.65 & 20.26 & 23.08 & 23.23 & 18.91 & 17.89 \\
\hline BSE & 10 & 1600 & 10 & 27.55 & 26.86 & 25.13 & 22.67 & 19.6 & 20.71 & 19.94 & 20.83 & 20.29 & 18.59 & 20.61 & 20.75 & 17.78 & 17.05 \\
\hline BSE & 10 & 2000 & 10 & 25.43 & 24.76 & 23.29 & 21.27 & 18.77 & 19.79 & 18.97 & 19.95 & 18.87 & 17.96 & 19.56 & 19.75 & 17.3 & 16.59 \\
\hline $\mathrm{CC}$ & 100 & 1200 & 30 & 32.14 & 30.63 & 30.77 & 29.08 & 26.7 & 20.88 & 29.5 & 21.49 & 23.1 & 21.39 & 30.22 & 21.46 & 19.15 & 16.98 \\
\hline $\mathrm{CC}$ & 100 & 1600 & 30 & 31.27 & 30.01 & 30.22 & 28.46 & 25.98 & 20.65 & 29.0 & 21.27 & 23.0 & 21.23 & 29.87 & 21.24 & 18.93 & 16.88 \\
\hline $\mathrm{CC}$ & 100 & 2000 & 30 & 27.21 & 26.42 & 26.15 & 25.06 & 20.93 & 19.09 & 22.43 & 19.52 & 20.29 & 18.89 & 23.85 & 19.48 & 16.92 & 16.15 \\
\hline $\mathrm{CC}$ & 10 & 1200 & 30 & 29.43 & 28.48 & 28.76 & 27.54 & 23.44 & 19.93 & 25.48 & 20.44 & 21.41 & 19.71 & 26.5 & 20.38 & 17.55 & 16.37 \\
\hline $\mathrm{CC}$ & 10 & 1600 & 30 & 26.06 & 25.31 & 24.7 & 23.72 & 19.63 & 18.82 & 20.73 & 19.08 & 19.28 & 18.11 & 21.94 & 19.06 & 16.5 & 15.76 \\
\hline $\mathrm{CC}$ & 10 & 2000 & 30 & 23.33 & 22.59 & 21.24 & 19.65 & 17.1 & 17.97 & 17.56 & 17.86 & 17.38 & 16.5 & 18.19 & 17.91 & 15.76 & 15.2 \\
\hline BSE & 100 & 1000 & 30 & 36.56 & 34.76 & 32.83 & 28.57 & 21.74 & 22.35 & 22.36 & 22.52 & 23.44 & 19.71 & 23.21 & 22.77 & 18.1 & 16.94 \\
\hline BSE & 100 & 1600 & 30 & 29.16 & 28.4 & 26.62 & 23.57 & 19.4 & 20.7 & 19.89 & 20.71 & 21.21 & 18.32 & 20.66 & 20.93 & 17.21 & 16.41 \\
\hline BSE & 100 & 2000 & 30 & 26.41 & 25.82 & 24.11 & 21.69 & 18.48 & 19.58 & 18.86 & 19.72 & 19.43 & 17.55 & 19.57 & 19.7 & 16.66 & 15.95 \\
\hline BSE & 10 & 1000 & 30 & 30.43 & 29.51 & 27.74 & 24.41 & 19.83 & 21.11 & 20.35 & 21.14 & 21.82 & 18.64 & 21.13 & 21.42 & 17.41 & 16.54 \\
\hline BSE & 10 & 1600 & 30 & 25.01 & 24.47 & 22.85 & 20.69 & 17.94 & 19.01 & 18.23 & 19.14 & 18.45 & 17.08 & 18.87 & 19.04 & 16.34 & 15.65 \\
\hline BSE & 10 & 2000 & 30 & 23.08 & 22.51 & 21.2 & 19.45 & 17.16 & 18.13 & 17.34 & 18.32 & 17.2 & 16.51 & 17.89 & 18.11 & 15.89 & 15.22 \\
\hline $\mathrm{CC}$ & 100 & 2200 & 10 & 28.12 & 27.23 & 26.95 & 26.04 & 21.71 & 20.25 & 23.01 & 20.66 & 21.07 & 19.76 & 24.38 & 20.6 & 17.94 & 17.16 \\
\hline $\mathrm{CC}$ & 10 & 2200 & 10 & 24.47 & 23.62 & 22.29 & 20.65 & 18.16 & 19.12 & 18.58 & 18.97 & 18.33 & 17.54 & 19.17 & 19.0 & 16.88 & 16.34 \\
\hline BSE & 100 & 2200 & 10 & 27.9 & 27.17 & 25.43 & 22.93 & 19.76 & 20.87 & 20.12 & 20.97 & 20.55 & 18.71 & 20.81 & 20.93 & 17.87 & 17.14 \\
\hline BSE & 10 & 2200 & 10 & 24.62 & 23.94 & 22.61 & 20.76 & 18.43 & 19.45 & 18.59 & 19.61 & 18.38 & 17.74 & 19.14 & 19.37 & 17.12 & 16.42 \\
\hline $\mathrm{CC}$ & 100 & 2200 & 30 & 25.54 & 24.78 & 23.94 & 22.9 & 19.01 & 18.72 & 19.95 & 18.87 & 18.79 & 17.73 & 21.0 & 18.88 & 16.35 & 15.61 \\
\hline $\mathrm{CC}$ & 10 & 2200 & 30 & 22.16 & 21.43 & 20.24 & 18.74 & 16.54 & 17.48 & 16.9 & 17.37 & 16.69 & 16.01 & 17.44 & 17.4 & 15.45 & 14.98 \\
\hline BSE & 100 & 2200 & 30 & 25.33 & 24.77 & 23.12 & 20.91 & 18.08 & 19.13 & 18.4 & 19.28 & 18.67 & 17.17 & 19.05 & 19.19 & 16.4 & 15.72 \\
\hline BSE & 10 & 2200 & 30 & 22.35 & 21.75 & 20.57 & 18.98 & 16.85 & 17.81 & 16.98 & 17.99 & 16.77 & 16.3 & 17.5 & 17.76 & 15.72 & 15.07 \\
\hline
\end{tabular}




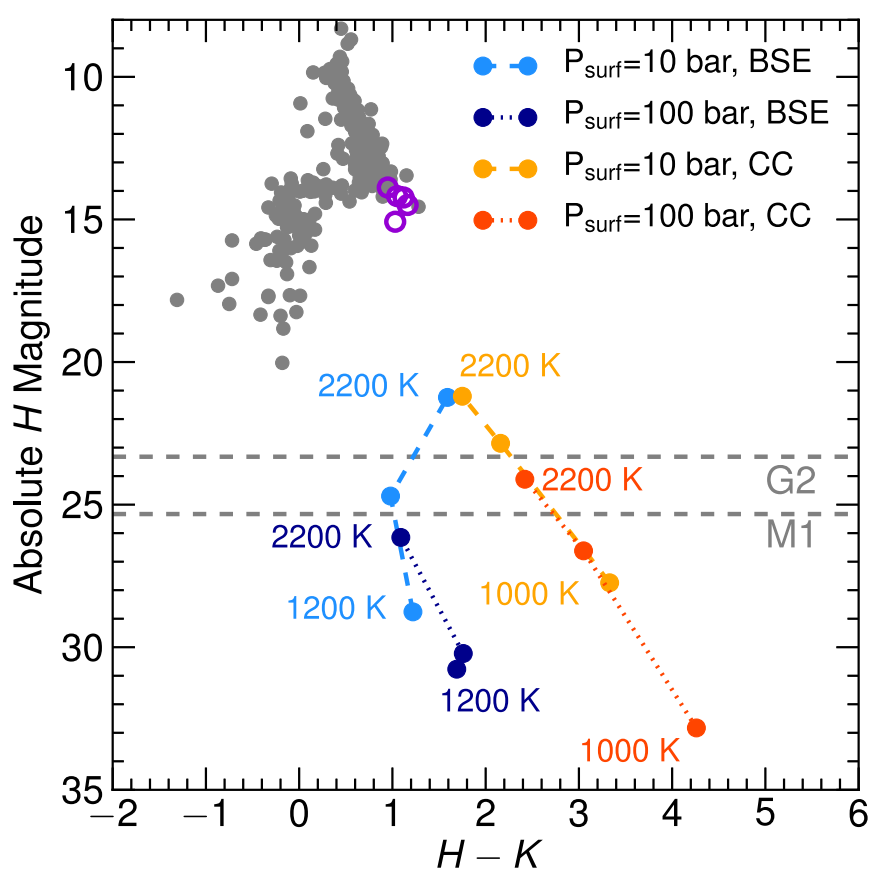

Figure 14. Color-magnitude diagram of postimpact Earths. $H-K$ color vs. absolute $H$ magnitude for a selection of models. The continental crust composition atmosphere are shown in blue, and the bulk silicate Earth models are shown in red. Two surface pressures (10 and 100 bar) are shown; all models have surface gravities of $30 \mathrm{~m} \mathrm{~s}^{-2}$. Observed brown dwarfs are shown as gray circles; directly imaged planets (4 HR 8799 planets, Skemer et al. 2012; Marois et al. 2010 and 2M1207b, Patience et al. 2010) are shown as violet open circles. Estimated magnitude limits for a planet around a G2 and M1 dwarf observed with a $30 \mathrm{~m}$ class telescope capable of $10^{-8}$ contrast are shown as dashed gray lines; models above the line should be observable with a $30 \mathrm{~m}$ telescope. The magnitudes and colors for the post-giant-impact planets are computed using the equilibrium chemistry. After correcting for vertical mixing and photochemistry, the BSE points would move closer to the CC models.

(A color version of this figure is available in the online journal.)

$\left(t_{\text {chem }}>t_{\text {dyn }}\right)$. Chemical equilibrium is not attained in this disequilibrium region, and the chemical equilibrium abundances established at the quench level prevail in the absence of further disequilibration due to photochemistry. While a complete analysis of disequilibrium chemistry for our atmospheric models is beyond the scope of this paper, we can identify possible deviations from chemical equilibrium and their effect on the predicted spectra.

Following Gierasch \& Conrath (1985), we calculate the vertical eddy diffusion coefficient $K_{z z}$ from mixing length theory as a function of height in the atmosphere (or equivalently pressure):

$$
K_{z z}=\frac{1}{3} h_{z}\left(\frac{R_{\mathrm{gas}} \dot{Q}_{c z}}{\mu_{z} \rho_{z} c_{p z}}\right)^{1 / 3}
$$

where $h_{z}$ is the scale height, $R_{\text {gas }}$ is the ideal gas constant, $\mu_{z}, \rho_{z}$, and $c_{p z}$ are the mean molecular weight, density, and specific heat capacity of the gas, $\dot{Q}_{c z}$ is the convective heat flow, and the index $z$ is used to designate that these quantities vary with height. The convective heat flow $\dot{Q}_{c z}$ is calculated as the difference between the total atmospheric energy flux $\left(\sigma_{B} T_{\text {eff }}^{4}\right)$ and the integrated radiative flux at any particular position in the atmosphere, as given by our converged model. The dynamical timescale is then $t_{\text {dyn }}=h_{z}^{2} / K_{z z}$. We find that for the hottest models, which are most likely to be observed, the dynamical timescale for mixing from the tropopause to the surface varies between 2 and $20 \mathrm{hr}$ for the BSE and between 1 and $15 \mathrm{hr}$ for the CC case. Thus, the gas will be mixed and in contact with the hot magma present at the surface on a day-like timescale.

The bulk of the atmosphere for the $\mathrm{CC}$ and BSE compositions consists of $\mathrm{H}_{2} \mathrm{O}$ and $\mathrm{CO}_{2}$, which are found throughout the atmosphere. Other species, such as $\mathrm{CH}_{4}$ and $\mathrm{NH}_{3}$, which play an important role especially in the BSE models, are most abundant at low temperatures, and their abundances can more easily be affected by disequilibrium chemistry. For these two species, we consider the kinetics of the reactions:

$$
\begin{aligned}
& \mathrm{CH}_{4}+\mathrm{OH} \rightarrow \mathrm{CH}_{3}+\mathrm{H}_{2} \mathrm{O} \\
& k_{1}=2.6 \times 10^{-17}\left(T^{1.83}\right) \exp (-1400 / T),\left[\mathrm{cm}^{3} \mathrm{~s}^{-1}\right]
\end{aligned}
$$

and

$$
\begin{aligned}
& \mathrm{NH}_{3}+\mathrm{OH} \rightarrow \mathrm{NH}_{2}+\mathrm{H}_{2} \mathrm{O} \\
& k_{2}=8.31 \times 10^{-17}\left(T^{1.6}\right) \exp (-480 / T),
\end{aligned}
$$

with the corresponding reaction rates $k_{1}$ and $k_{2}$. The rate constants for reactions 2 and 3 are from Baulch et al. (1992) and Cohen \& Westberg (1991), respectively. A comparison of the $t_{\text {chem }}$ and $t_{\text {dyn }}$ values shows that both reactions quench in the 900-1000 K range for the CC and BSE model atmospheres. As a result, the atmospheric abundances of $\mathrm{CH}_{4}$ and $\mathrm{NH}_{3}$ at lower temperatures are frozen in at their values at the 900-1000 K isothermal region in the atmosphere. These abundances are negligible (Figures 4 and 5); therefore, the equilibrium abundance curves will no longer be valid (dashed lines). The kinetic inhibition of $\mathrm{CH}_{4}$ and $\mathrm{NH}_{3}$ formation also means that the $\mathrm{CO}_{2}$ and $\mathrm{N}_{2}$ abundance curves are quenched at their high temperature values, i.e., the abundances of $\mathrm{CO}_{2}$ and $\mathrm{N}_{2}$ are not reduced by the formation of significant amounts of methane and ammonia. Consequently, since $\mathrm{N}_{2}$ is not an important absorber, $\mathrm{CO}_{2}$ will replace $\mathrm{CH}_{4}$ and $\mathrm{NH}_{3}$ as the dominant opacity source after water.

\subsection{Photochemistry}

We use a new one-dimensional atmospheric photochemistry code that is a hybrid between a "hot Jupiter" photochemistry code (Zahnle et al. 2009; Miller-Ricci Kempton et al. 2012) and a conventional terrestrial code (Zahnle et al. 2006; Claire et al. 2006). Vertical mixing is parameterized by the eddy diffusion coefficient, $K_{z z}$, which is determined by the atmosphere structure model (Section 4.1). The new code currently includes about 1100 reactions of 96 small species (molecules and free radicals) made from $\mathrm{H}, \mathrm{O}, \mathrm{C}, \mathrm{N}, \mathrm{S}, \mathrm{Na}$, and $\mathrm{Cl}$. Missing from the model are species of $\mathrm{F}$ and $\mathrm{K}$, and the important omissions are likely to be $\mathrm{HF}$ and $\mathrm{KCl}$. Every chemical reaction in the photochemistry code is balanced by the corresponding reverse reaction, with the reverse reaction rate determined by thermodynamic equilibrium (TE). This ensures that the kinetics model will relax to thermochemical equilibrium in the absence of vertical mixing and radiation. The model includes $\mathrm{H}_{2} \mathrm{O}, \mathrm{H}_{2} \mathrm{SO}_{4}, \mathrm{~S}_{8}, \mathrm{NaCl}$, $\mathrm{NaOH}, \mathrm{NaCN}$, and $\mathrm{Na}_{2} \mathrm{~S}$ aerosols. The aerosols condense, fall, and evaporate, but chemistry on grain surfaces is not included. Nor have we yet to fully implement dissolution of species or aerosols into cloud droplets.

The right-hand panels in Figures 4 and 5 show the results of the photochemistry calculation for a $1600 \mathrm{~K}$ surface and a 100 bar atmosphere, for atmospheres that at the surface are in equilibrium with $\mathrm{CC}$ or BSE, respectively. Two things stand out: (1) vertical mixing is very important in these atmospheres, and (2) photochemistry is important at the top. The kinetically 

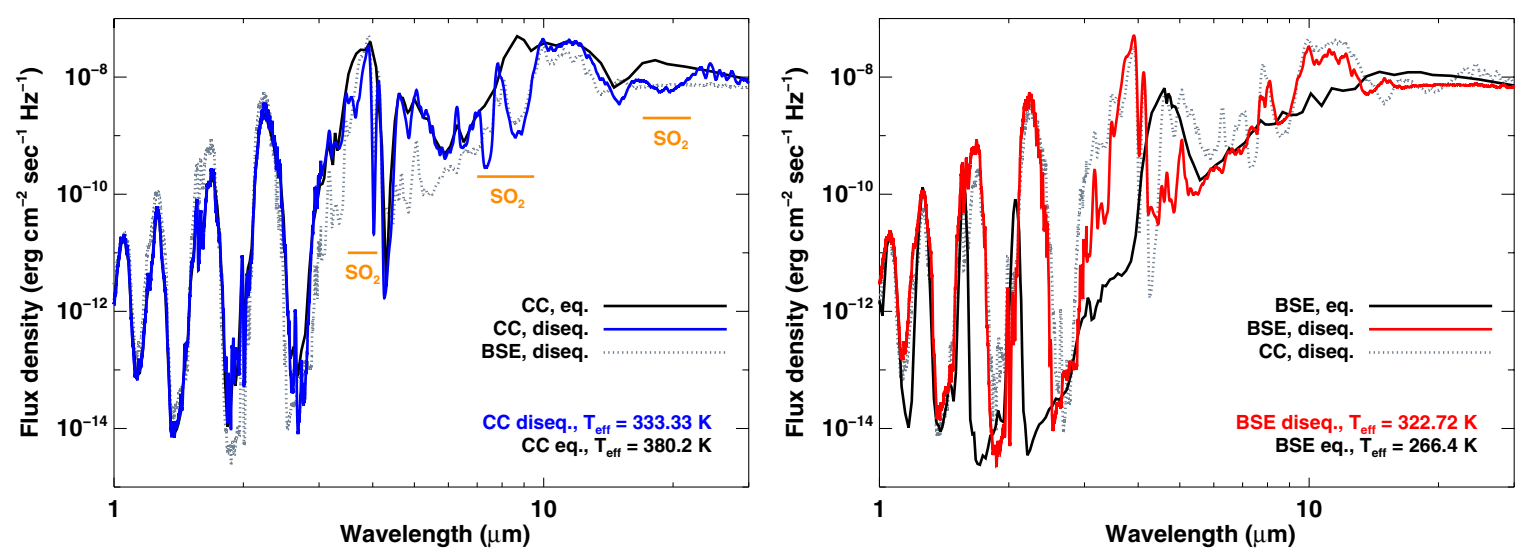

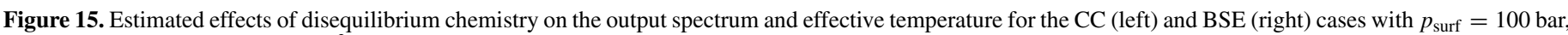

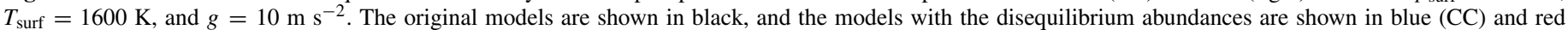

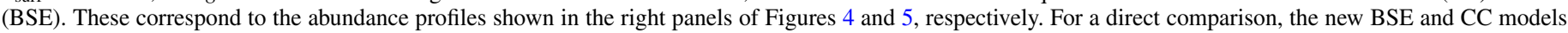

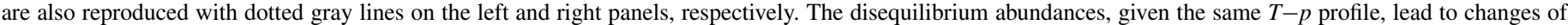
about $50 \mathrm{~K}$ in the effective temperatures.

(A color version of this figure is available in the online journal.)

computed atmospheres in the IR will broadly resemble the emission from the atmospheres in TE at $\sim 1000 \mathrm{~K}$ ( 5 bars). Several species are seen to mix to high altitude, in abundances far in excess of the predictions of TE. Interesting examples include $\mathrm{H}_{2} \mathrm{~S}$ and other sulfur species in the BSE atmosphere, $\mathrm{O}_{2}$, and more $\mathrm{SO}_{2}$ in the $\mathrm{CC}$ atmosphere. Other molecules that TE predicts might be abundant are barely seen $\left(\mathrm{CH}_{4}\right.$ and $\mathrm{NH}_{3}$ are the most important); their absence was expected from quenching as discussed above.

A notable difference between the equilibrium chemistry code and the kinetics code is that the kinetics code predicts that sulfur species should extend throughout the atmosphere. The high abundance of $\mathrm{SO}_{2}$ in the $\mathrm{CC}$ case is especially noteworthy. The different sulfur species conform roughly to their relative TE abundances at $1000 \mathrm{~K}$, although the absolute abundances are much greater. This is likely to be correct in a general sense; the TE assumption that sulfur species from cool parts of the atmosphere will condense as, for example, $\mathrm{CaSO}_{4}$ at the surface is not very realistic. The kinetics code also predicts that $\mathrm{HCl}$ vapor and $\mathrm{NaCl}$ crystals (halite) would be mixed to high altitudes. These predictions are more likely to be artifacts because the model does not yet account for $\mathrm{HCl}$ and $\mathrm{NaCl}$ dissolving in water clouds. On the other hand, the $\mathrm{HCl}$ abundance is so high that it may exceed what can be accommodated by clouds. HF, which we have not computed, is also likely to stay in the gas phase to high altitudes given the high strength of the HF bond.

The detailed kinetics predictions in Figures 4 and 5 for $\mathrm{CH}_{4}$ and $\mathrm{NH}_{3}$ agree well with predictions made with the quenching approximation, described in Section 4.1. Since most other relevant species are sulfur compounds, identifying the relevant reactions and the corresponding timescales for these species may make it faster and easier to explore the parameter space of possible atmospheric compositions over magma oceans.

To explore the impact disequilibrium chemistry might have on the emergent spectrum, we computed the high-resolution spectra (Figure 15) for the BSE and CC $p_{\text {surf }}=100 \mathrm{bar}$ and $g=10 \mathrm{~m} \mathrm{~s}^{-2}$ cases by using the disequilibrium chemical abundances to replace the equilibrium values. The original models are shown in black, while the alternate composition is shown dotted on both plots, for comparison (CC versus BSE). Since we do not converge a new thermal profile, the models now have a new emergent flux and $T_{\text {eff }}$, which are also indicated on the plots. Especially for the BSE atmosphere (right panel), the spectra show significant differences in spectral morphology; thus, the effect of disequilibrium chemistry in these atmospheres would be discernible in even low resolution spectra. For the CC case, the $\mathrm{SO}_{2}$ spectral features become even more prominent. As apparent from the right panel, after taking into account the chemical disequilibrium in the atmosphere, the spectra for the BSE and CC compositions become similar because of the predominance of water and $\mathrm{CO}_{2}$ in both cases. The BSE model is distinguishable in this case by additional $\mathrm{CO}$ absorption.

We stress that the photochemical models are not yet fully selfconsistent radiative-convective atmosphere models, since they are computed for a fixed atmospheric structure. The computed effective temperature of the altered models is larger in the BSE case without the methane and ammonia opacity and is lower for the $\mathrm{CC}$ atmosphere because of extra $\mathrm{SO}_{2}$ opacity. A fully self-consistent model would have to properly account for the effect of mixing as the model converges, which is beyond the scope of this preliminary investigation. The removal of $\mathrm{CH}_{4}$ and $\mathrm{NH}_{3}$ from the $\mathrm{CC}$ models does not yield significant spectral changes, since the abundances of these compounds were still low in the original models. It is interesting to note, however, that the spectral signatures of $\mathrm{HF}, \mathrm{HCl}$, and $\mathrm{SO}_{2}$ should remain present regardless of assumptions about mixing, as the spectral features for these molecules are formed in the hotter parts of the atmosphere that are expected to be in chemical equilibrium. In fact, for the $\mathrm{CC}$ composition the $\mathrm{SO}_{2}$ features become some of the most prominent, even for 100 bar atmospheres (Figure 15, left), because of the increase in total column for this important absorber.

\section{DISCUSSION}

\subsection{Evolution Timescales}

The opacity of the atmosphere will be one of the determining factors for the time evolution of the planet's temperature after the giant impact. Understanding this cooling process is also important for characterizing the formation of the Earth-Moon system, which is still subject to many uncertainties in the current models. As shown in Figure 3, even when the surface is at $2200 \mathrm{~K}$ because of the presence of greenhouse gasses in the 

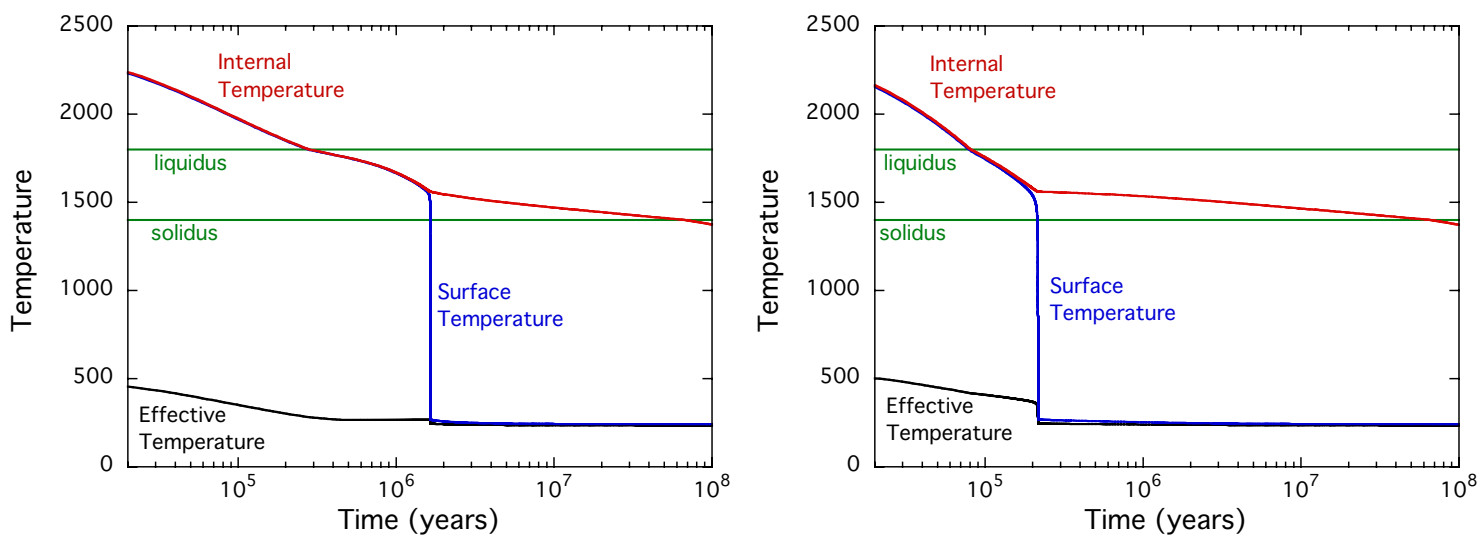

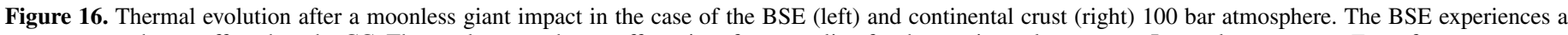

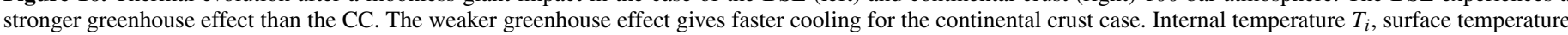

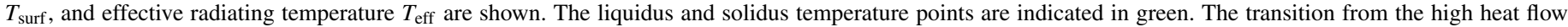

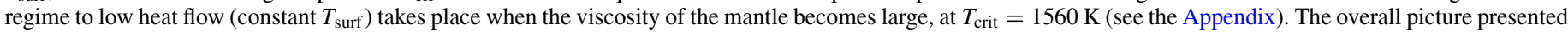
here closely follows that first discussed by Abe (1993).

(A color version of this figure is available in the online journal.)

atmosphere, the planet only radiates at an effective temperature of $500-750 \mathrm{~K}$ for the $\mathrm{CC}$ or $450-800 \mathrm{~K}$ for the BSE, depending on surface pressure. This limits the amount of heat that can be lost by the planet per unit time.

We consider the generic case of an earthlike planet after a giant impact that does not form a Moon. We follow the evolution in time of this planet, starting with a hot molten magma planetary surface at a temperature of $2400 \mathrm{~K}$, which gradually cools and solidifies. The postimpact atmosphere is assumed to have a surface pressure of 100 bar, which remains constant until the planetary mantle completely solidifies. The liquidus is set at $1800 \mathrm{~K}$ (above which the mantle is purely liquid), while the solidus is set at $1400 \mathrm{~K}$ (below which the mantle is completely solid). In between these two temperatures, the mantle is only partially solidified. The heat stored in the mantle at any given temperature is a function of the heat capacity of the mantle and the fraction of material in liquid state. Assuming a convective interior, the energy flow from the mantle to the surface is governed by the Rayleigh number of the fluid, a function of temperature, viscosity, thermal diffusivity, and expansion coefficient. The total energy radiated by the planet is equivalent to that of a blackbody with a temperature equal to $T_{\text {eff }}$ (which also includes insolation). The full derivation of the cooling process, as well as the relevant quantities, are given in the Appendix. The particular case where the giant impact results in the formation of a satellite such as the Earth's Moon, considering the tidal interaction and orbit evolution of the two bodies, will be addressed in a future paper.

Figure 16 shows the results of our calculations for an earthlike planet. The interior, surface, and radiating (effective) temperatures are shown in red, blue, and black, respectively. The BSE model with $p_{\text {surf }}=100$ bar (left) has a stronger greenhouse and spends more time above the liquidus. Even though in such a scenario the surface of the planet stays hot for a longer time, the low effective temperature will make it harder to detect than a planet with a more transparent atmosphere (such as the CC model). The sharp transition in the surface temperature marks the point where the mantle begins to solidify and its viscosity becomes large (1560 K, see the Appendix). As the interior temperature drops below this value, the heat transfer from the interior to the surface becomes small, and the surface temperature is solely determined by the energy balance with the solar radiation. Therefore, the surface temperature does not change, giving rise to the sharp transition in Figure 16, the breadth of which is determined by the relatively tiny heat capacity of the atmosphere. This analysis shows that the models discussed in this work are applicable to a timeframe spanning $\sim 10^{7} \mathrm{yr}$ after the planet has undergone a giant impact. The planet will be in a hot state and therefore more easily detectable for a time period of $\sim 10^{5} \mathrm{yr}$ after the impact. Finally, we note that in view of the discussion regarding disequilibrium chemistry, the BSE atmosphere will be more transparent, while the CC atmosphere will be more opaque than used in this calculation. We expect that this change will result in a shorter (longer) cooling timescale for the BSE (CC) cases, in which case the evolution for the two atmospheric compositions will resemble each other more closely. Nevertheless, the BSE equilibrium composition can be considered as a relevant case for a planet undergoing a runaway greenhouse stage during its cooling process.

\subsection{Observing Post-giant-impact Earths}

\subsubsection{Detection}

As discussed in Section 3.4, despite their high surface temperatures, postimpact Earths will have relatively low effective temperatures; this will make them extremely challenging to observe. The observational limit is primarily due to the ability to suppress the host star's light with adaptive optics and a coronagraph. The first "extreme" adaptive optics systems on $8 \mathrm{~m}$ class telescopes (the Gemini Planet Imager (GPI) SPHERE) will achieve $\sim 10^{-7}$ contrast at angular separations greater than 100 mas; for a sun-like star observed in the $H$ band, this corresponds to an absolute $H$ magnitude of $\sim 21$. This means that the hottest models in this study $\left(p_{\text {surf }}=10\right.$ bar and $\left.T_{\text {surf }}=2200 \mathrm{~K}\right)$ will be at the edge of the reachable contrast limit, in contrast to the models of Miller-Ricci et al. (2009), which estimate a contrast between $10^{-6}$ and $10^{-7}$ for a $1 M_{\oplus}$ planet with similar surface pressure and temperature and place Earth-mass planets with $p_{\text {surf }}=100 \mathrm{bar}$ and $T_{\text {surf }}=1500 \mathrm{~K}$ at the limit of detectability.

For comparison, in Figure 14 we show the observed brown dwarfs (gray circles) and directly imaged exoplanets (purple circles). The brightest of our models lie at about 6 mag fainter in $H$ band than the currently imaged extrasolar planets, namely 2M1207b (Patience et al. 2010) and the planets around HR 8799 
(Skemer et al. 2012; Marois et al. 2010). However, following the trend of $H$-band magnitude with surface pressure (lighter versus darker symbols), postimpact Earths with tenuous atmospheres ( $\sim 1$ bar) would fall several magnitudes closer to the HR 8799 planets because of the hot surface being less obscured. If at an appropriate angular separation, such planets would be in the detectability range of the $8 \mathrm{~m}$ class telescopes but over a shorter timescale, since they would cool much faster than a young giant planet. Such observations would test the theories of volatile-poor accretion during terrestrial planet formation, as these nearly volatile-free hot Earths would be the brightest among similar size planets for a brief period of time.

A $30 \mathrm{~m}$ class telescope with extreme adaptive optics will be capable of an order of magnitude higher contrast; Macintosh et al. (2006) estimate that this class of telescope will be capable of $10^{-8}$ contrast at $1.65 \mu \mathrm{m}$ at angular separations of tens of milliarcseconds. For a sun-like G2 dwarf, this corresponds to a $H$-band absolute magnitude of 23.3 ; for a fainter M1 dwarf, the limiting magnitude is 25.3. Figure 14 shows these magnitude limits; many of the hot postimpact Earths fall above these lines, suggesting that they will be observable with a $30 \mathrm{~m}$ class telescope, especially if cooler stars are targeted.

Following Stern (1994), the average number of stars we would need to search to find one post-giant-impact earthlike planet is $N_{*}=1 / N_{t} /\left(t_{\text {acc }} / t_{\text {hot }}\right)$, where $N_{t}$ is the number of terrestrial planets per star, $t_{\mathrm{acc}}$ is the timespan of the giant impact era during terrestrial planet formation, and $t_{\text {hot }}$ is the length of time the planet stays hot during the entire period of giant impacts. The latest estimates (Bonfils et al. 2013; Kopparapu et al. 2013) give an occurrence rate of about $0.4-0.6$ terrestrial planets in the habitable zone, per star. According to terrestrial planet formation models (Wetherill 1992; Agnor et al. 1999), $t_{\mathrm{acc}}$ is in the range 40-100 Myr. Melis et al. (2010) have shown that the giant impact stage, as constrained by the presence of warm dust, occurs between 30 to 100 Myr for solar-mass stars and much earlier, between 10 and $30 \mathrm{Myr}$, for stars of a few solar masses. The time the planet is hot after each giant impact is about $3 \times 10^{5} \mathrm{yr}$ (see Figure 16). Stewart \& Leinhardt (2012) find that it takes on average between 10 and 16 giant impacts to make an earthlike planet. Therefore, the total $t_{\text {hot }}$ in the best-case scenario will be $16 \times 3 \times 10^{5}$ yr. Therefore, the number of young stars to be observed in order to find a post-giant-impact earthlike planet is about 14 in the best-case scenario. This number becomes $\sim 40$ in a not-so-favorable region of the parameter space.

There are a number of nearby $(<50 \mathrm{pc})$ young stellar clusters in the age range where giant impacts would be expected among the young terrestrial planets. Examples include the Tucana-Horologium association (age 10-30 Myr, with about 50 stars at 50 pc; Zuckerman et al. 2001) and the closer AB Dor moving group (age $~ 50 \mathrm{Myr}$, about 30 stars at $20 \mathrm{pc}$; Zuckerman et al. 2004). The $\beta$ Pic moving group, the TW Hydrae association, and the $\eta$ Cha cluster are all younger and include more stars. There are clearly a sufficient number of nearby young stars to motivate a survey for post-giant-impact planets. This estimate is in agreement with previous calculations by Miller-Ricci et al. (2009) and Jackson \& Wyatt (2012), who find that about $10 \%$ of the stars could host such a post-giant-impact planet. One of the goals of future surveys will be precisely to constrain the number of stars hosting such post-giant-impact planets (i.e., $N_{*}$ as defined above) and to narrow the parameter space currently in use by the terrestrial planet formation models. We emphasize that our estimate takes into account only terrestrial planets the hab- itable zone. The number of possible detections could increase by factors of a few if we consider planets outside this zone, as well as non-earthlike planets.

\subsubsection{Moons and Disks}

Another direct consequence of a giant impact is the scattering of debris, with the formation of a circumstellar debris disk, and the possible formation of a moon. Observing a newly formed moon is unlikely. The probability of forming a moon is $\sim 8 \%$ (Elser et al. 2011). Although the moon is formed hot and potentially much brighter because of the lack of atmosphere, its surface cooling time is comparatively short relative to the planet, about 400 yr (e.g., Pritchard \& Stevenson 2000). On the basis of the same argument as above, the number of stars one would need to observe in order to find such a moon would be on the order of $10^{4}$. It is clearly more likely that future surveys will detect the post-giant-impact planet, even with a cooler $T_{\text {eff }}$, rather than the moon, which only forms in $8 \%$ of the cases.

However a giant impact will not only melt the target but will also likely create a disk of impact-generated debris surrounding the star in the orbit of the target planet. Such a disk might both be a signature of the impact and a hindrance for efforts at direct imaging in the near-IR. The degree to which this is true depends on the evolution of the disk, including effects of Poynting-Robertson drag and gap clearing by the planet itself. Indeed, the observational signature of an impact-generated debris disk is an area of active research as part of the extrasolar zodiacal light (exozodi) issue, which affects planet detectability in general (e.g., Roberge et al. 2012; Turnbull et al. 2012). A detailed study of the signature of such a disk is well beyond the scope of this paper, but we present a few notional calculation to estimate its effect in order to provide a comparison to the estimated flux of the planet.

In general, the direct detection of any planet at optical wavelengths will require a space-based mission, so we are primarily concerned with scattered light and thermal emission from the disk at IR wavelengths. The post-giant-impact debris will cool to a temperature of $\sim 278 \mathrm{~K}$ (at $1 \mathrm{AU}$ ) on a 100-1000 yr timescale (Jackson \& Wyatt 2012; Kains et al. 2011). Thermal emission from such a disk will peak in the mid-IR. Depending on the size of the largest fragment, the postimpact debris disk luminosity $10^{5}-10^{6} \mathrm{yr}$ after the impact can be about $10^{-3}-10^{-7}$ that of the star. The lower limit is equal to the unit of 1 zodi. Since currently planned missions aim for levels of 10 zodi or less for detecting earthlike planets, we use the 1-10 zodis range as the scaling for the integrated luminosity of the disk. Levels as high as 100 zodis would also be acceptable, coupled with a significant increase in exposure time (e.g., Turnbull et al. 2012). Figure 10 shows that the radiation from the post-giant-impact planet will exceed the integrated thermal radiation from the dust (green dot-dashed line) at wavelengths shorter than about $\sim 4 \mu \mathrm{m}$. With a $30 \mathrm{~m}$ class telescope the disk will be resolved, and the light from the disk will be spread out over a large number of pixels, increasing the contrast between the disk and the planet. The flux density from the debris disk in Figure 10 is calculated per resolution element (equal to a diffraction-limited circle at all wavelengths), assuming the dust has a circular distribution 0.8 AU wide, centered at $1 \mathrm{AU}$ from the star. The post-giant-impact system is placed 100 lt-yr (about $30 \mathrm{pc}$ ) away from the observer.

Virtually nothing is known about the exozodi scattering properties of disks around young stars, since most debris disks are discovered in the far-IR. Assuming a constant optical albedo 
of 0.12 (similar to the Moon's) and using the same luminosity scaling as above (1 and 10 zodis), the scattered light from the disk is shown in blue in Figure 10. In this case, the scattered light from the disk can be larger than the emission from the planet in the visible, but we find that the radiation from the 10 bar atmosphere could be stronger than a 10 zodi background in the $2-4 \mu \mathrm{m}$ range. It is apparent from the same figure that the water windows below $4 \mu \mathrm{m}$ would offer the best contrast for detecting the post-giant-impact planets. The detection of IR excess at 10-20 $\mu \mathrm{m}$ can signal the presence of a debris disk, and such stars can be then followed up at shorter wavelengths (1-4 $\mu \mathrm{m})$ with high resolution adaptive optics in order to detect potential post-giant-impact planets. The possible complication from a brighter debris disk can be dealt with observationally. If the planet is imaged directly, some light from the disk will fall into the planet's point-spread function, but this can be efficiently subtracted as a background by using the spectral energy distribution measured by combining the extra pixels occupied by the disk alone. In this case, the contribution from the disk will not affect the atmospheric signatures but will likely add noise to the spectrum. We refer the reader to Roberge et al. (2012) and Turnbull et al. (2012) for a more rigorous calculation and in-depth description of these issues.

\section{CONCLUSIONS}

We have investigated the atmospheres of earthlike planets following a giant impact event. As discussed in more detail by Schaefer et al. (2012), the chemical compositions considered, BSE and CC, characterize a class of rocky planets around stars of solar or near-solar metallicity, with a differentiated metal $+\mathrm{FeS}$ bearing core and a silicate exterior and with all $\mathrm{Fe}$ as $\mathrm{Fe}^{2+}$ and $\mathrm{Fe}^{3+}$ in minerals. These post-giant-impact atmospheres are composed of vapors in equilibrium with the magma ocean at the bottom and of gases rising through turbulent mixing at altitudes where the temperature drops below 900-1000 K. The main constituents, dominating the energy transport through the atmosphere, are water and $\mathrm{CO}_{2}$. We find that the postimpact chemistry also produces characteristic molecules, namely $\mathrm{HCl}$, $\mathrm{HF}$, alkali halides, $\mathrm{SO}_{2}$, and other sulfur-bearing species that are not expected in cooler atmospheres. By contrast, anhydrous worlds have no volatiles and thus no $\mathrm{HF}$ and $\mathrm{HCl}$ (Schaefer \& Fegley 2009). We also note the presence of significant amounts of $\mathrm{Na}$ and $\mathrm{K}$ in these atmospheres, with potentially detectable [ I] $\mathrm{Na}$ ] lines for thinner (10 bar) atmospheres. The spectral signatures of these species can thus be considered as markers for post-giant-impact earthlike planets. However, the spectral resolution and contrast needed for such observations is still beyond the capabilities of current instrumentation for exoplanet characterization. Nevertheless, the near-IR colors of such worlds are expected to be notably redder than young giant planets and may thus be at least tentatively identified on this basis.

The blanketing effect of the atmosphere obscures the hot surface of postimpact planets at IR wavelengths. For surface temperatures between 1000 and $2200 \mathrm{~K}$, the corresponding effective temperatures are found to be only as high as $\sim 700 \mathrm{~K}$ for an earthlike planet with $p_{\text {surf }}=100$ bar and possibly as low as $265 \mathrm{~K}$ when the planet is completely in a runaway greenhouse state. These low effective temperatures, as well as the addition of more opacity sources that diminish the escape of radiation through the $\mathrm{H}_{2} \mathrm{O}$ and $\mathrm{CO}_{2}$ windows, make the post-giant-impact planets in these models less bright than previously thought (e.g., Miller-Ricci et al. 2009). Because of the added opacity sources, the brightness temperature in the water windows is decreased by factors of two to three. This translates into flux densities that are far fainter in the spectral windows than predicted by Miller-Ricci et al. (2009), depending on the wavelength. In the case of thin, tenuous atmospheres $(\sim 1$ bar), or larger surface gravities, some of these planets may still be detectable with $8 \mathrm{~m}$ class telescopes. However, for the higher surface pressure cases discussed in this paper, even the hottest planets will only be accessible to $30 \mathrm{~m}$ class telescopes.

On the basis of the heat transport from the planet's interior and through the atmosphere, we estimate that these post-giantimpact planets will stay hot on the order of one million years and may undergo several such impacts during their formation. As an outcome of a giant impact, the hot planet is definitely more likely to be observed than a potential young moon. In the 1-4 $\mu \mathrm{m}$ wavelength region, sufficient contrast may also be achieved to distinguish such a planet from a surrounding debris disk. Given the observational capabilities, it is thus plausible that some planets will be found in this state by direct imaging with coronagraphs on large telescopes. On the basis of the expected frequency of terrestrial planets, we estimate that one such postgiant-impact planet may be discovered for every 10 to 50 stars surveyed in young stellar clusters.

Our models do not yet include opacities from clouds and aerosols, which may further diminish the predicted brightness temperatures in the IR. Disequilibrium chemistry also proves to be important for these atmospheres, and a self-consistent approach, including vertical mixing, photochemistry, and clouds, to the calculation of the radiative-convective atmosphere is the subject of future work.

This work was supported by the NASA Origins program. B.F. was supported by the NSF Astronomy Program, the NASA EPSCOR Program, and by NASA Cooperative Agreement NNX09AG69A with the NASA Ames Research Center.

\section{APPENDIX}

We describe magma ocean cooling by taking into account convective heat flow through the mantle, the heat capacities of the mantle and core, the heat of fusion when the mantle congeals, and the thermal blanketing effect imposed by the atmosphere. Plausibly important factors that we do not include here are high heat flow from a superheated core, tidal heating by a nearby moon or moons, tidal heating by the sun, and if very early, heating by decay of ${ }^{26} \mathrm{Al}$. We will address tidal heating by a moon elsewhere. For simplicity, we treat the interior as characterized by a single potential temperature $T_{i}$, the temperature that any parcel in an adiabatic mantle would have were it brought to the surface, while the surface temperature itself is $T_{\text {surf }}$.

We describe silicate freezing by three temperatures: a liquidus $T_{\text {liq }}$ above which the mantle is fully molten; a solidus $T_{\text {sol }}$, below which the mantle is fully solid; and a critical temperature $T_{\text {crit }}$ in between where the rheology of the material changes from that of a solid with melt percolating through it to a liquid in which solids are suspended (Abe 1997; Solomatov 2007). We take $T_{\text {liq }}=1800 \mathrm{~K}$ and $T_{\text {sol }}=1400 \mathrm{~K}$ and assume that the melt fraction $\phi$ is linear in $T_{i}$,

$$
\phi= \begin{cases}1, & T_{i}>T_{\text {liq }} \\ \left(T_{i}-T_{\text {sol }}\right) /\left(T_{\text {liq }}-T_{\text {sol }}\right), & T_{\text {sol }}<T_{i}<T_{\text {liq }} \\ 0, & T_{i}<T_{\text {sol }}\end{cases}
$$


For $T_{\text {liq }}<T_{i}<T_{\text {sol }}$, we approximate the heat capacity of our post-giant-impact planet with that of the Earth, $C M_{\oplus}=$ $C_{\mathrm{v}} M_{\text {man }}+C_{\mathrm{c}} M_{\text {core }}=6.2 \times 10^{34} \mathrm{erg} \mathrm{g}^{-1} \mathrm{~K}^{-1}$, in which the heat capacity of silicate is $C_{\mathrm{v}}=1.2 \times 10^{7} \mathrm{erg} \mathrm{g}^{-1} \mathrm{~K}^{-1}$ and for the iron core is $C_{\mathrm{c}}=6.5 \times 10^{6} \mathrm{erg} \mathrm{g}^{-1} \mathrm{~K}^{-1}$. The iron core does not freeze on the timescale of interest. While silicates freeze, the effective heat capacity of the Earth is

$$
C^{\prime} M_{\oplus}=C M_{\oplus}+\frac{Q_{\mathrm{m}} M_{\mathrm{man}}}{T_{\mathrm{liq}}-T_{\mathrm{sol}}}=1.0 \times 10^{34} \mathrm{erg} \mathrm{K}^{-1},
$$

where the heat of fusion is $Q_{\mathrm{m}}=4 \times 10^{9} \mathrm{erg} \mathrm{g}^{-1}$, and $M_{\operatorname{man}}$ is the mass of the mantle, $M_{\operatorname{man}}=4.1 \times 10^{27} \mathrm{~g}$.

Following Solomatov (2007), we treat mantle cooling by parameterized convection. In this framework, heat flow is related to the size and viscosity of the interior and the temperature gradient across the surface boundary layer through the Rayleigh number Ra:

$$
F=C_{0} \frac{k_{\mathrm{c}}\left(T_{i}-T_{\text {surf }}\right)}{L} \mathrm{Ra}^{n},
$$

where $C_{0}=0.089$ is a constant (Solomatov 2007), $k_{\mathrm{c}}=$ $3 \times 10^{5} \mathrm{erg} \mathrm{cm}^{-2} \mathrm{~s}^{-1} \mathrm{~K}^{-1}$ is the thermal conductivity, and $L$ is a characteristic distance usually identified with the thickness of the mantle $\left(L=2.8 \times 10^{8} \mathrm{~cm}\right)$. The power is $n=1 / 3$ in soft turbulence (Solomatov 2007). The Rayleigh number describes the ratio of buoyancy to viscosity,

$$
\mathrm{Ra}=\frac{\alpha_{\mathrm{v}} g\left(T_{i}-T_{\text {surf }}\right) L^{3}}{\kappa v} .
$$

In Equation (A4), $\alpha_{\mathrm{v}}=2.4 \times 10^{-5} \mathrm{~K}^{-1}$ is the volume thermal expansivity, $g$ the gravity, $\kappa=0.01 \mathrm{~cm}^{2} \mathrm{~s}^{-1}$ is the thermal diffusivity $\left(=k_{\mathrm{c}} / C_{\mathrm{v}} / \rho\right)$, and $v$ the kinematic viscosity $\left(\mathrm{cm}^{2} \mathrm{~s}^{-1}\right)$.

Viscosity is a strong function of temperature. Following Abe (1997), Solomatov (2007), and Lebrun et al. (2013), we divide viscosity regimes into a rheological liquid and a rheological solid. In the rheological liquid, Solomatov parameterizes viscosity by

$$
v=6 \times 10^{-4} \exp \left(\frac{4600}{T_{i}-1000}\right)\left(\frac{1-\phi_{\text {crit }}}{\phi-\phi_{\text {crit }}}\right)^{2.5} .
$$

This expression for $v \rightarrow \infty$ as $\phi \rightarrow \phi_{\text {crit }}$; i.e., it is singular at the critical value $\phi=\phi_{\text {crit }}$. In the rheological solid,

$$
v=1 \times 10^{19} \exp \left(\frac{T_{\text {sol }}-T_{i}}{58}\right) \exp \left(-\alpha_{\phi} \phi\right),
$$

where $26<\alpha_{\phi}<32$ (Solomatov 2007). Abe (1997) places the boundary at $\phi=\phi_{\text {crit }}=0.4$; the precise value of $\phi_{\text {crit }}$ is not very important. For $\phi_{\text {crit }}=0.4$, the critical temperature for the rheological transition is $T_{\text {crit }}=1560 \mathrm{~K}$.

Equations (A3) and (A4) can be combined to give

$$
F=C_{0} k_{\mathrm{c}}\left(T_{i}-T_{\text {surf }}\right)^{(1+n)} L^{(3 n-1)}\left(\frac{\alpha_{\mathrm{v}} g}{\kappa v}\right)^{n} .
$$

Usually, $n=1 / 3$ is used, in which case $L$ cancels out. This gives a system in which the heat flow is determined only by the properties of the boundary layer, which is often regarded as the physically meaningful case.

The cooling of the planet interior is described by

$$
\begin{aligned}
C M_{\oplus} \frac{d T_{i}}{d t}=-4 \pi R_{\oplus}^{2} F & T_{i}>T_{\mathrm{liq}}, T_{i}<T_{\mathrm{sol}} ; \\
C^{\prime} M_{\oplus} \frac{d T_{i}}{d t}=-4 \pi R_{\oplus}^{2} F & T_{\mathrm{sol}}<T_{i}<T_{\text {liq }} .
\end{aligned}
$$

Taking into account the incoming solar flux, $F_{\text {sun }}$, and the total heat capacity of the atmosphere, $C_{\mathrm{A}}\left(\mathrm{erg} \mathrm{g}^{-1} \mathrm{~K}^{-1}\right)$, the global planetary cooling rate is described by

$$
\begin{aligned}
& C M_{\oplus} \frac{d T_{i}}{d t}+C_{\mathrm{A}} \frac{d T_{\text {surf }}}{d t}=-4 \pi R_{\oplus}^{2} \sigma T_{\mathrm{eff}}^{4}+\pi R_{\oplus}^{2} F_{\text {sun }}(1-A) \\
& T_{i}>T_{\text {liq }}, T_{i}<T_{\text {sol }} \\
& C^{\prime} M_{\oplus} \frac{d T_{i}}{d t}+C_{\mathrm{A}} \frac{d T_{\text {surf }}}{d t}=-4 \pi R_{\oplus}^{2} \sigma T_{\mathrm{eff}}^{4}+\pi R_{\oplus}^{2} F_{\text {sun }}(1-A) \\
& T_{\text {sol }}<T_{i}<T_{\text {liq }}
\end{aligned}
$$

The net radiative cooling is determined by the effective radiating temperature of the atmosphere, taking into account the sunlight absorbed, where $F_{\text {sun }} \approx 1 \times 10^{6} \mathrm{erg} \mathrm{cm}^{-2} \mathrm{~s}^{-1}$ was the solar constant ca. $4.5 \mathrm{Ga}$, and $A$ is the planetary albedo $(A=0.3$ today). The effective radiating temperature $T_{\text {eff }}$ is determined by the thermal blanketing of the atmosphere and the surface temperature $T_{\text {surf }}$. The relation between $T_{\text {eff }}$ and $T_{\text {surf }}$ is derived from fits to the curves in Figure 3, for $1000<T_{\text {surf }}<2200 \mathrm{~K}$. These curves are derived for equilibrium chemistry. After taking into account vertical mixing (Section 4), we expect that the curves for the BSE and CC cases will resemble each other more closely.

Equations (A8) and (A9) are solved for the evolution of $T_{i}$, $T_{\text {surf }}$, and $T_{\text {eff }}$. Figure 16 shows possible thermal evolutions of an earthlike planet after a giant impact that does not produce a Moon.

\section{REFERENCES}

Abe, Y. 1993, GMS, 74, 41

Abe, Y. 1997, PEPI, 100, 27

Abe, Y., \& Matsui, K. 1988, JAtS, 45, 3081

Agnor, C., \& Asphaug, E. 2004, ApJL, 613, L157

Agnor, C. B., Canup, R. M., \& Levison, H. F. 1999, Icar, 142, 219

Allard, F., Hauschildt, P. H., \& Schwenke, D. 2000, ApJ, 540, 1005

Alvarez, R., \& Plez, B. 1998, A\&A, 330, 1109

Baulch, D. L., Cobos, C. J., Cox, R. A., et al. 1992, JPCRD, 21, 411

Benz, W., Slattery, W. L., \& Cameron, A. G. W. 1986, Icar, 66, 515

Berdyugina, S. V., \& Livingston, W. C. 2002, A\&A, 387, L6

Bezard, B., de Bergh, C., Crisp, D., \& Maillard, J.-P. 1990, Natur, 345, 508

Bonfils, X., Delfosse, X., Udry, S., et al. 2013, A\&A, 549, A109

Brown, L. 2005, JQSRT, 96, 251

Burrows, A., Marley, M. S., \& Sharp, C. M. 2000, ApJ, 531, 438

Burrows, A., Ram, R. S., Bernath, P., Sharp, C. M., \& Milsom, J. A. 2002, ApJ, 577,986

Cameron, A. G. W., \& Ward, W. R. 1976, in Lunar and Planetary Institute Science Conference Abstracts, Vol. 7 (Houston, TX: LPI), 120

Canup, R. M. 2004, Icar, 168, 433

Canup, R. M., \& Asphaug, E. 2001, Natur, 412, 708

Canup, R. M., \& Righter, K. 2000, in Origin of the Earth and Moon, ed. W. K. Hartmann, R. J. Phillips, \& G. J. Taylor (Houston, TX: LPI)

Chambers, J. E. 2004, E\&PSL, 223, 241

Claire, M. W., Catling, D. C., \& Zahnle, K. J. 2006, Geobiology, 4, 239

Cohen, N., \& Westberg, K. R. 1991, JPCRD, 20, 1211

Cushing, M. C., Marley, M. S., Saumon, D., et al. 2008, ApJ, 678, 1372

Dana, V., Mandin, J.-Y., Guelachvili, G., et al. 1992, JMoSp, 152, 328

Dulick, M., Bauschlicher, C. W., Jr., Burrows, A., et al. 2003, ApJ, 594, 651

Elser, S., Moore, B., Stadel, J., \& Morishima, R. 2011, Icar, 214, 357

Fegley, B., \& Prinn, R. G. 1988, ApJ, 326, 490

Fegley, B., Jr., \& Lodders, K. 1994, Icar, 110, 117

Fegley, B., Jr., \& Lodders, K. 1996, ApJL, 472, L37

Fegley, B., Jr., \& Prinn, R. G. 1985, ApJ, 299, 1067

Fegley, B., Jr., \& Prinn, R. G. 1986, ApJ, 307, 852

Fegley, B., Jr., \& Schaefer, L. 2012, Chapter 13.3 in Treatise on Geochemistry ed. H. D. Holland \& K. K. Turekian (Amsterdam: Elsevier)

Fortney, J. J., Lodders, K., Marley, M. S., \& Freedman, R. S. 2008a, ApJ, 678,1419

Fortney, J. J., Marley, M. S., Saumon, D., \& Lodders, K. 2008b, ApJ, 683,1104 
Freedman, R. S., Marley, M. S., \& Lodders, K. 2008, ApJS, 174, 504

Gamache, R. R., Lynch, R., \& Neshyba, S. P. 1998, JQSRT, 59, 319

Gierasch, P., \& Goody, R. 1968, P\&SS, 16, 615

Gierasch, P. J., \& Conrath, B. J. 1985, in Recent Advances in Planetary Meterology, ed. G. E. Hunt (Cambridge: Cambridge Univ. Press), 121

Goldblatt, C., Crisp, D., Robinson, T., Watson, A., \& Zahnle, K. 2012, LPICo, 1675,8095

Goody, R., West, R., Chen, L., \& Crisp, D. 1989, JQSRT, 42, 539

Hamano, K., Abe, Y., \& Genda, H. 2013, Natur, 497, 607

Hargreaves, R. J., Hinkle, K. H., Bauschlicher, C. W., Jr., et al. 2010, AJ, 140,919

Harris, G. J., Larner, F. C., Tennyson, J., et al. 2008, MNRAS, 390, 143

Harris, G. J., Tennyson, J., Kaminsky, B. M., Pavlenko, Y. V., \& Jones, H. R. A. 2006, MNRAS, 367, 400

Harrison, T. M., Blichert-Toft, J., Müller, W., et al. 2005, Sci, 310, 1947

Hartmann, W. K., Phillips, R. J., \& Taylor, G. J. (ed.) 1986, Origin of the Moon

Hashimoto, G. L., Abe, Y., \& Sugita, S. 2007, JGRE, 112, 5010

Ingersoll, A. P. 1969, JAtS, 26, 1191

Jackson, A. P., \& Wyatt, M. C. 2012, MNRAS, 425, 657

Kains, N., Wyatt, M. C., \& Greaves, J. S. 2011, MNRAS, 414, 2486

Kargel, J. S., \& Lewis, J. S. 1993, Icar, 105, 1

Kasting, J. F. 1988, Icar, 74, 472

Kissel, A., Sumpf, B., Kronfeldt, H.-D., Tikhomirov, B., \& Ponomarev, Y. 2002, JMoSp, 216, 345

Kopparapu, R. K., Ramirez, R., Kasting, J. F., et al. 2013, ApJ, 765, 131

Kurucz, R. L. 2011, CaJPh, 89, 417

Langhoff, S. R., \& Bauschlicher, C. W., Jr. 1993, CPL, 211, 305

Lattanzi, F., di Lauro, C., \& Vander Auwera, J. 2011, JMoSp, 267, 71

Lebrun, T., Massol, H., Chassefire, E., et al. 2013, JGRE, 118, 1155

Lewis, J. S. 1969, Icar, 10, 365

Lissauer, J. J. 1993, ARA\&A, 31, 129

Lodders, K., \& Fegley, B., Jr. 2006, Chemistry of Low Mass Substellar Objects, ed. J. W. Mason (Chichester: Praxis), 1

Macintosh, B., Troy, M., Doyon, R., et al. 2006, Proc. SPIE, 6272, 62720N

Marley, M. S., Gelino, C., Stephens, D., Lunine, J. I., \& Freedman, R. 1999, ApJ, 513,879

Marley, M. S., \& McKay, C. P. 1999, Icar, 138, 268

Marley, M. S., Saumon, D., Guillot, T., et al. 1996, Sci, 272, 1919

Marois, C., Zuckerman, B., Konopacky, Q. M., Macintosh, B., \& Barman, T. 2010, Natur, 468, 1080

Matsui, T., \& Abe, Y. 1986, Natur, 319, 303

Melis, C., Zuckerman, B., Rhee, J. H., \& Song, I. 2010, ApJL, 717, L57

McKay, C. P., Pollack, J. B., \& Courtin, R. 1989, Icar, 80, 23

Mehrotra, S. C., \& Nair, K. P. R. 1981, JChPh, 75, 2479

Miller-Ricci, E., Meyer, M. R., Seager, S., \& Elkins-Tanton, L. 2009, ApJ, 704,770

Miller-Ricci Kempton, E., Zahnle, K., \& Fortney, J. J. 2012, ApJ, 745, 3

Moynier, F., Yin, Q. Z., Irisawa, K., et al. 2010, PNAS, 107, 10810

Nemtchinov, V., Sung, K., \& Varanasi, P. 2004, JQSRT, 83, 243

Nikitin, A. V., Holka, F., Tyuterev, V. G., \& Fremont, J. 2009, JChPh, 130,244

Partridge, H., \& Schwenke, D. W. 1997, JChPh, 106, 4618

Patience, J., King, R. R., de Rosa, R. J., \& Marois, C. 2010, A\&A, 517, A76

Pavlov, A. A., Kasting, J. F., Brown, L. L., Rages, K. A., \& Freedman, R. 2000, JGR, 105, 11981

Pollack, J. B., \& Ohring, G. 1973, Icar, 19, 34

Prinn, R. G., \& Barshay, S. S. 1977, Sci, 198, 1031
Pritchard, M. E., \& Stevenson, D. J. 2000, in Origin of the Earth and Moon, ed R. M. Canup, K. Righter et al. (Tucson, AZ: Univ. Arizona Press), 179

Ramanathan, V., \& Coakley, J. A. 1978, Rev. Geophys. Space Phys., 16, 465

Raymond, S. N., Quinn, T., \& Lunine, J. I. 2004, Icar, 168, 1

Richard, C., Gordon, I., Rothman, L., et al. 2012, JQSRT, 113, 1276

Roberge, A., Chen, C. H., Millan-Gabet, R., et al. 2012, PASP, 124, 799

Rothman, L., Gordon, I., Barber, R., et al. 2010, JQSRT, 111, 2139

Rothman, L. S., Gordon, I. E., Barbe, A., et al. 2009, JQSRT, 110, 533

Saumon, D., \& Marley, M. S. 2008, ApJ, 689, 1327

Schaefer, L., \& Fegley, B. 2009, ApJL, 703, L113

Schaefer, L., \& Fegley, B. 2010, Icar, 208, 438

Schaefer, L., \& Fegley, B., Jr. 2011, ApJ, 729, 6

Schaefer, L., Lodders, K., \& Fegley, B. 2012, ApJ, 755, 41

Schwenke, D. W. 1998, FaDi, 109, 321

Skemer, A. J., Hinz, P. M., Esposito, S., et al. 2012, ApJ, 753, 14

Solomatov, V. 2007, in Treatise on Geophysics, ed. G. Schubert (Amsterdam: Elsevier), 91

Stamnes, K., Tsay, S.-C., Jayaweera, K., \& Wiscombe, W. 1988, ApOpt, 27,2502

Stern, S. A. 1994, AJ, 108, 2312

Stewart, S. T., \& Leinhardt, Z. M. 2012, ApJ, 751, 32

Strong, K., Taylor, F. W., Calcutt, S. B., Remedios, J. J., \& Ballard, J. 1993, JQSRT, 50, 363

Tipping, R. 1976, JMoSp, 61, 272

Toon, O. B., McKay, C. P., Ackerman, T. P., \& Santhanam, K. 1989, JGR, 94, 16287

Toth, R. A., Hunt, R. H., \& Plyler, E. K. 1970, JChPh, 53, 4303

Turnbull, M. C., Glassman, T., Roberge, A., et al. 2012, PASP, 124, 418

van Zeggeren, F., \& Storey, S. H. 1970, The Computation of Chemical Equilibria (Cambridge: Cambridge Univ. Press)

Wattson, R., \& Rothman, L. 1992, JQSRT, 48, 763

Wattson, R. B., \& Rothman, L. S. 1986, JMoSp, 119, 83

Weber, M. 2003, Handbook of Optical Materials, The CRC Press Laser and Optical Science and Technology Series (Washington, DC: CRC)

Weck, P. F., Schweitzer, A., Kirby, K., Hauschildt, P. H., \& Stancil, P. C. 2004, ApJ, 613, 567

Weck, P. F., Schweitzer, A., Stancil, P. C., Hauschildt, P. H., \& Kirby, K. 2003a, ApJ, 582, 1059

Weck, P. F., Stancil, P. C., \& Kirby, K. 2003b, JChPh, 118, 9997

Wedepohl, K. H. 1995, GeCoA, 59, 1217

Wenger, C., \& Champion, J. P. 1998, JQSRT, 59, 471

Wetherill, G. W. 1985, Sci, 228, 877

Wetherill, G. W. 1992, in Lunar and Planetary Institute Workshop on the Physics and Chemistry of Magma Oceans from 1 Bar to 4 Mbar, ed. C. B. Agee \& J. Longhi (Houston, TX: LPI), 74

Wilde, S. A., Valley, J. W., Peck, W. H., \& Graham, C. M. 2001, Natur, 409, 175

Yurchenko, S. N., Barber, R. J., \& Tennyson, J. 2011, MNRAS, 413, 1828

Zahnle, K., Arndt, N., Cockell, C., et al. 2007, SSRv, 129, 35

Zahnle, K., Claire, M., \& Catling, D. 2006, Geobiology, 4, 271

Zahnle, K., Marley, M. S., Freedman, R. S., Lodders, K., \& Fortney, J. J. 2009, ApJL, 701, L20

Zahnle, K. J. 2006, Elements, 2, 217

Zahnle, K. J., Kasting, J. F., \& Pollack, J. B. 1988, Icar, 74, 62

Zare, R., Schmeltekopf, A., Harrop, W., \& Albritton, D. 1973, JMoSp, 46, 37

Zuckerman, B., Song, I., \& Bessell, M. S. 2004, ApJL, 613, L65

Zuckerman, B., Song, I., \& Webb, R. A. 2001, ApJ, 559, 388 